\title{
Differentially expressed Inc - NOS2P3-miR-939-5p axis in chronic heart failure inhibits myocardial and endothelial cells apoptosis via iNOS/TNFa pathway
}

Cuncun Chen ( $\sim$ ccc121501@126.com)

Tongji University Affiliated Shanghai Eastern hospital https://orcid.org/0000-0001-9473-7289

\section{Ming Zong}

Tongji University Affiliated Shanghai Eastern Hospital

\section{Ying Lu}

Tongji University Affiliated Shanghai Eastern hospital

\section{Yide Guo}

Tongji University Affiliated Shanghai Eastern hospital

\section{Honggen Lv}

Tongji University Affiliated Shanghai Eastern hospital

\section{Lihong Xie}

Tongji University Affiliated Shanghai Eastern hospital

\section{Zhiyan Fu}

Tongji University Affiliated Shanghai Eastern hospital

\section{Yu Cheng}

Tongji University Affiliated Shanghai Eastern hospital

\section{Yuying Si}

Tongji University Affiliated Shanghai Eastern Hospital

\section{Bei Ye}

Tongji University Affiliated Shanghai Eastern Hospital

\section{Lieying Fan}

Tongji University Affiliated Shanghai Eastern Hospital

\section{Original investigation}

Keywords: LncRNA-NOS2P3, MicroRNA-939-5p, INOS, TNFa, Apoptosis, Chronic heart failure

Posted Date: March 11th, 2020

DOI: https://doi.org/10.21203/rs.3.rs-16823/v1 
License: (c) (i) This work is licensed under a Creative Commons Attribution 4.0 International License. Read Full License

Version of Record: A version of this preprint was published at Journal of Cellular and Molecular Medicine on August 25th, 2020. See the published version at https://doi.org/10.1111/jcmm.15740. 
Differentially expressed lnc-NOS2P3-miR-939-5p axis in chronic heart failure inhibits myocardial and endothelial cells apoptosis via iNOS/TNF $\alpha$ pathway

Cuncun Chen, * Ming Zong, Ying Lu, Yide Guo, Honggen Lv, Lihong Xie, Zhiyan Fu, Yu Cheng, Yuying Si, Bei Ye, Lieying Fan *

Department of Clinical Laboratory, Shanghai East Hospital, Tongji University School of Medicine, 150 Ji Mo Road, Shanghai 200120, People's Republic of China

${ }^{*}$ Correspondence to:

Prof. Lieying Fan or Dr. Cuncun Chen

Shanghai East Hospital, Tongji University School of Medicine, 150 Ji Mo Road, Shanghai 200120, People's Republic of China.

Phone: +86-21-38804518. Fax: +86-21-58798999

E-mail: flieying@yeah.net or ccc121501@,126.com 


\section{Abstract}

\section{Background:}

Inflammatory cytokines induced cells apoptosis is important for initiation and progression of chronic heart failure (CHF). Long noncoding RNAs (lncRNAs) and microRNAs (miRNAs) were critical in this pathogenesis. However, their roles in inflammation and apoptosis of CHF remain unclear.

\section{Methods:}

A total of $75 \mathrm{CHF}$ and 36 non-CHF control patients were collected and the level of miR-939-5p was detected. Bioinformatics analysis and luciferase reporter assay was used to predict and verify pairs of IncRNA-miRNA-mRNA. RT-qPCR was used to evaluate the expression of IncRNA, miRNAs and mRNAs. CCK8 and flow cytometry were applied to determine cells vitality and apoptosis. Western blot was used for protein level analysis.

\section{Results:}

CHF patients had elevated serum miR-939-5p, with greater increase in NYHA I-II patients than in NYHA III-IV. Moreover, miR-939-5p was positively correlated with B-type natriuretic peptide (BNP) in NYHA III-IV patients, while not in New York Heart Association (NYHA) I-II, suggesting that miR-939-5p might be an additional supplement for diagnosis of heart failure. Further study showed miR-939-5p mimic promoted proliferation and inhibited inflammatory cytokines induced apoptosis of HUVECs and H9C2, while inhibition of endogenous miR-939-5p by antagomir produced the opposite effects. INOS and TNF a were identified and confirmed as 
target genes of miR-939-5p. Moreover, we identified lncRNA-NOS2P3 as a sponge RNA to inhibit miR-939-5p expression, regulate the expression of iNOS/TNF a , and control inflammation induced myocardial and endothelial cells apoptosis.

\section{Conclusions:}

Together, CHF patients exhibited elevated serum miR-939-5p especially in NYHA I-II grades. Lnc-NOS2P3-miR-939-5p-iNOS/TNF a pathway played an important role in regulating inflammatory cytokines induced cells apoptosis and provided a promising strategy for diagnosis and treatment of CHF.

Key words: LncRNA-NOS2P3; MicroRNA-939-5p; INOS; TNFa; Apoptosis;

Chronic heart failure 


\section{Background}

As more and more patients survive their initial cardiovascular diseases, chronic heart failure (CHF) becomes the leading cause of elderly hospitalization and death worldwide [1]. Therapeutic strategies improvements didn't increase CHF survival rate effectively [2]. CHF is a progressive disease, and the critical pathogenesis is myocardial pathological remodeling, which is initially triggered by multiple signal and network interactions [3] including overactivation of many neurohormones and inflammation factors after myocardial injury $[4,5]$. CHF is classified into four grades according to ESC guidelines, diagnosis and treatment in early grades would accept more ideal therapeutic effect [6].

Circulating inflammatory cytokines predict clinical outcomes, and dynamically changed during the process of CHF. The apoptosis induced by inflammatory cytokines of myocardial and endothelial cells (ECs) facilitates myocardial pathological remodeling [5]. After heart injury, the main method to improve regenerative capacity is to stimulate cardiomyocyte proliferation [7]. Cardiomyocyte proliferation rate increases after injury, but it remains insufficient to fully regenerate the lost myocardial tissue [8]. Exception for myocytes, non-myocyte population of heart consists of $>60 \%$ endothelial cells [9] presenting in a much higher frequency have direct implications on cardiac pathophysiology [10]. Inducing only myocyte proliferation after MI (Myocardial infarction, MI) would cause defective heart regeneration with newly formed myocyte unlikely to survive in the absence of ECs [11]. Fast revascularization is essential to support cardiac regeneration [12]. And the regeneration ends 
prematurely in the injured area without vascularization [13]. To inhibit inflammation induced myocardial and endothelial cells apoptosis in CHF is critical.

A large number of non-coding RNAs will be activated during development of CHF, including microRNAs (miRNAs) and long non-coding RNAs (lncRNAs) [14]. MicroRNAs (miRNAs), 19-25 nucleotides, modulate the physiological and pathological process in transcriptional or post-transcriptional levels and could be induced by inflammation which could regulate myocardial diseases. MiR-155 containing in macrophage exosomes suppressed fibroblast proliferation and promote fibroblast inflammation during cardiac injury [15]. The miR-146b-TRAF6-IL-6/CCL2 (MCP-1) axis drives cardiac inflammation, fibrosis and ventricular dysfunction [16]. Inhibition of miR-103 attenuates inflammation and endoplasmic reticulum stress in atherosclerosis through disrupting the PTEN-mediated MAPK signaling [17]. But there is little study about inflammation related miRNAs and heart failure. LncRNAs, exceeding 200 nucleotides, were reported to be closely related to the regulation of CHF [18], and function as competing RNAs of miRNAs to regulate cardiac remodeling through transcriptional and posttranscriptional levels [19, 20].

Normal heart maintains low level of nitric oxide (NO) synthesized from $\mathrm{Ca} 2+$ dependent endothelial nitric oxide synthase (eNOS), which inhibits apoptosis of myocardial and endothelial cells to preserve cardiac normal physiology. However, inflammation activated $\mathrm{Ca} 2+$ independent inducible nitric oxide synthase (iNOS) will produce high level of NO, leads to apoptosis of myocardial and endothelial cells, and causes negative inotropic effect [21], and NO level in CHF patients was much higher 
[22]. More, inflammatory TNF $\alpha$ was reported to promote iNOS production in mice [23]. Further study found slightly increased level of iNOS and TNF $\alpha$ in NYHA I-II grades CHF patients with no significance compared with normal controls, but was significantly up-regulation in NYHA III-IV patients [24]. To specifically inhibit NO from iNOS and maintain its balance in CHF is essential. Guo et al. identified iNOS as target gene of miR-939-5p in hepatocytes [25]. And our microarray analysis also indicated iNOS may be its candidate target.

Our previous study selected 11 miRNAs including miR-939-5p to predict the early virological response to interferon treatment in chronic hepatitis $\mathrm{B}$ (CHB) patients [26]. The expression of miR-939-5p in hepatic perforation tissues of CHB patients was positively correlated with ALT level $(\mathrm{R}=0.42, p<0.05)$, and in immune activation group (ALT $>40 \mathrm{U} / \mathrm{L}$ ) was higher than that in immune tolerance group (ALT $<40 \mathrm{U} / \mathrm{L}$ ) (data not shown). Meanwhile, miRNA-939-5p restricted Hepatitis B virus by targeting Jumonji Domain Containing 3 (JMJD3) mediated chromatin remodeling [27]. Moreover, we found exsomes secreted from liver non-parenchymal cells contained large amount of miR-939-5p when stimulated by IFN- $\alpha$ [28]. The activation of retinoic acid-induced gene protein I (RIG-I) pathway also produced a large amounts of miRNA-939-5p [29]. Taken together, miR-939-5p was extensively related to chronic inflammation. However, there is no study between miR-939-5p and CHF. This study we explored miR-939-5p expression in CHF patients and found serum miR-939-5p level in CHF patients elevated obviously compared to non-CHF, with greater level in NYHA I-II grades than NYHA III-IV. And miR-939-5p was positively 
correlated with BNP in NYHA III-IV patients, while not in NYHA I-II. Then we found miR-939-5p inhibited the inflammatory cytokines induced myocardial and endothelial cells apoptosis. Moreover, miR-939-5p inhibited target genes iNOS and TNF $\alpha$ expression and the downstream NO synthesis. Further we identified lncRNA-NOS2P3 could act as an endogenous sponge RNA to repress miR-939-5p and its target genes expression and regulate its inhibition to inflammation induced cells apoptosis. Our research might provide a promising strategy for early diagnosis and treatment of chronic heart failure.

\section{Materials and Methods}

\section{Study population and data collection}

A total of $75 \mathrm{CHF}$ and 36 non-CHF control patients of Shanghai East hospital affiliated to Tongji University were collected from Oct. 2017 to Apr. 2018. There were no significant varieties in sex composition and age. CHF was defined according to ESC Guidelines of heart failure in 2016 [6] as Class I patients, no symptoms and no limitation in ordinary physical activity, e.g. shortness of breath when walking, climbing stairs etc; class II, mild symptoms and slight limitation during ordinary activity; Class III, marked limitation in activity due to symptoms, even during less-than-ordinary activity and Class IV, severe limitations, experiences symptoms even while at rest. 41 NYHA I-II patients and 24 NYHA III-IV patients were included in this study. Major exclusion criteria were patients with renal failure (serum creatinine $>176 \mu \mathrm{mol} / \mathrm{L}$ ); patients given digitalis and diuretics within 24-48 hours, and $\beta$-blockers used in the same day. 
Patients' fasting venous blood samples were obtained for BNP (analyzed by Roche cobas e411) and the patient's serum samples were stored at $-80^{\circ} \mathrm{C}$ for RNA isolation and analysis of miR-939-5p expression by quantitative reverse transcriptase polymerase chain reaction (RT-qPCR).

The Institutional Review Board of Tongji Medical School affiliated Shanghai East Hospital approved the study protocol and the written informed consent was obtained from each participant before any sample or data collection.

\section{Cell culture}

Human umbilical vein endothelial cells (HUVECs, ATCC® CRL-1730 ${ }^{\mathrm{TM}}$ ) were grown in RPMI 1640 medium (GIBCO, Grand Island, USA) supplemented with 10\% fetal bovine serum (FBS) (GIBCO, USA). H9C2 rat myocytes (ATCC, Rockvile, MD, USA) were cultured in Dulbecco's modified Eagle's medium (DMEM) (GIBCO, USA) with $10 \%$ FBS.

\section{RT-qPCR and microarray analysis}

Total RNA of serum samples and cultured cells was extracted by TRIzol (Life technologies). $600 \mu 1$ TRIzol was added into $300 \mu$ serum samples, and cel-miR-39 from RiboBio (Guangzhou, China) was added into the mixture as external reference. MicroRNAs were reverse transcribed using Bulge-loop RT primer and subsequently quantified with miRNA qPCR primer sets (both from RiboBio). LncRNAs were reverse transcribed using random primer and oligo dT primer. One Step SYBR ${ }^{\circledR}$ PrimeScript ${ }^{\circledR}$ PLUS RT-RNA PCR Kit (TaKaRa, Biotechnology) was used for first strand cDNA synthesis and quatiative PCR analysis. MiRNAs expression levels were 
normalized to U6. The expression of lncRNAs and mRNAs was normalized to GAPDH.

To identify target transcript and interacted lncRNAs of miRNA-939-5p, cells were transfected with miR-939 mimics $(100 \mathrm{nM})$ for $36 \mathrm{~h}$ and total RNA was harvested. CDNA microarray was performed using human LncRNA V2.0 from Arraystar Inc, which contains probes for protein coding transcripts and long non-coding RNAs, following the recommended workflow. Differentially expressed genes with over 2 fold change were selected for further filtering and RT-qPCR validation.

\section{Western blotting}

Total cell protein was extracted by RIPA lysis buffer (Beyotime. China), containing in protease inhibitors for $30 \mathrm{~min}$ at $4^{\circ} \mathrm{C}$. The protein concentration was determined using Bradford Assay (Bio-Rad). Total protein extracts of $20 \mu \mathrm{g}$ were resolved by SDS-PAGE gel electrophoresis and transferred to PVDF membranes (Bio-Rad) for western blot. Target protein was detected using specific primary antibody, and the primary antibodies were diluted as follows: $\mathrm{Bcl} 2$ (1:500, 2872S, cell-signalling), Bax (1:500, 5023S, cell-signalling), caspase3 (1:1000, SC7272, Santa Cruz), cyclinB1 (1:500, SC53236, Santa Cruz), iNOS (1:200, SC7271, Santa Cruz), $\beta$-actin (1:2000, SC47778, Santa Cruz). Bound antibodies were detected by a peroxidase-conjugated secondary antibody, and the signals were visualized using a chemiluminescence kit (Cell Signaling Technology). $\beta$-actin protein levels were used as loading control.

\section{Cell Transfection and treatment}

MiRNAs mimics, antagomirs, and lncRNA smart silencers (RiboBio, Guangzhou, 
China) were transfected into cells using lipofectamine 3000 (Life technologies) according to the manufacturer's instructions. The medium was changed into $1 \% \mathrm{FBS}$ RPI 1640/DMEM containing $2 \mu \mathrm{g} / \mathrm{ml}$ IL- $1 \beta$ and $800 \mathrm{IU} / \mathrm{ml}$ IFN- $\gamma 12$ hours later and cells would be collected for assays after treatment for 48 hours.

Lnc-NOS2P3 expression plasmids and its mutants of miR-939-5p conserved binding sites were constructed into pIRES2-EGFP plasmid (GENE, China), which were referred as lnc-NOS2P3-WT and lnc-NOS2P3-mut.

\section{CCK8 cell viability assay}

Cells were seeded in 48 -well culture plates and incubated at $37^{\circ} \mathrm{C}$ with $5 \% \mathrm{CO}_{2}$. After treatment, the CCK8 assay (Dojindo) was performed. Briefly, 1/10 volume CCK8 solution were added to each well and incubated for 1-2 hour. Then the supernatants were transferred to 96-well plate and the optical density of each well was measured at $450 \mathrm{~nm}$ with a microplate reader (Bio-Rad, USA).

\section{Apoptosis detection using Flow cytometry}

HUVECs or H9C2 myocytes were washed in PBS and resuspended at the density of 5 $\times 10^{5}$ cells $/ \mathrm{ml}$ with $500 \mu \mathrm{l}$ binding buffer $(1 \times)$. Afterwards, $5 \mu 1$ Annexin V-FITC solution and $5 \mu$ propidium iodide (BD, USA) were added into the suspension and the mixture were incubated for $15 \mathrm{~min}$ at room temperature avoiding light. Then, cells were performed with flow cytometry analysis.

\section{Dual-luciferase reporter assay}

TNF $\alpha-3$ 'UTR (799bp length) wide type containing miR-939-5p binding sites and its binding site mutation were cloned to pMIR-REPORT Luciferase H306 reporter 
plasmids, named as TNFa 3'UTR-WT/TNFa 3'UTR-MUT (provided by ObiO, Shanghai, China). The whole length of lnc-NOS2P3 and its mutant to miR-939-5p binding sites were constructed into GV272, named as lnc-NOS2P3-WT-luc and lnc-NOS2P3-mut-lnc (produced by GENECHEM, Shanghai, China).

HUVECs were seeded in 48 -well plate $\left(3 \times 10^{4}\right.$ per well $)$ and co-transfected with $0.5 \mu \mathrm{g}$

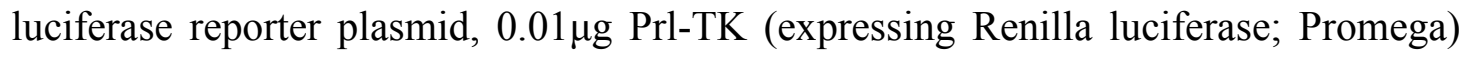
and 100nM miR-939-5p mimic or control. After 48 hours, cells were collected by passive lysis buffer and assayed for luciferase activity with Dual-Luciferase Assay kit (Promega). Firefly luciferase activities were normalized to renilla luciferase activities.

\section{Statistical analysis}

All data was analyzed using Graphpad Prism V5.0 and SPSS 18.0 statistical software and shown as standard error of the mean (SEM). Mann-Whitney U test was used to analyze miR-939-5p level in clinical samples. For cell culture experiments, student's $\mathrm{T}$ test was used. The correlation analysis was performed by Spearman's rank correlation. Data were considered significantly different if $p$-value $<0.05$.

\section{Results}

\section{Serum level of miR-939-5p in various NYHA grades of CHF patients}

To determine the role of miR-939-5p in CHF, we measured serum level of miR-939-5p in 75 CHF patients, with 41 NYHA I-II and 24 NYHA III-IV patients, compared with 36 non-CHF control patients. The results showed that CHF patients had significantly higher serum level of miR-939-5p than non-CHF control patients. 
And the level in NYHA I-II patients was obviously higher than in NYHA III-IV (Fig. 1A). Next, we analyzed the correlation between miR-939-5p and plasma B-type natriuretic peptide (BNP), the commonly marker for CHF diagnosis. No significant correlation was observed in NYHA I-II patients (Fig. 1B). But miR-939-5p has high correlation with BNP in NYHA III-IV, $\mathrm{r}=0.701, p<0.01$ (Fig. 1C). Usually, significant elevation of blood BNP suggests heart failure patients had entered into NYHA III or worse [2]. Meanwhile, BNP showed uncertainty in $100-400 \mathrm{pg} / \mathrm{ml}$ for diagnosis of CHF, especially with no significant clinical symptoms [30]. Serum miR-939-5p increased specifically in NYHA I-II patients and was not related to BNP. It showed different expression from BNP in CHF NYHA I-II patients, which may predict the possibility for early diagnosis of CHF. Of course, it needs further verification.

Previous study has demonstrated that miR-939-5p was an inflammation related miRNA [29, 31]. And in CHF progression, cytokines (such as TNF $\alpha$, IL-1 $\beta$, IL-6, galectin 3 and IFN- $\gamma$ ) were stimulated obviously [32].Taken together, miR-939-5p could be induced by inflammatory cytokines during CHF progression, especially in early stage of CHF.

MiR-939-5p inhibited inflammation induced apoptosis of endothelial cells HUVECs and myocardial cells $\mathrm{H} 9 \mathrm{C} 2$

Inflammatory cytokines induced cell apoptosis was the initial response to cardiac injury, which was important for cardiac remodeling contributing to CHF progression [4]. IL-1 $\beta$ and IFN- $\gamma$ have been shown to affect cardiac function by activating NO synthase activity in cardiac myocytes [33]. Myocardial and endothelial cells consist of 
majority of cardiac cells [9]. Thus in this study we investigate the regulation of miR-939-5p to inflammation induced apoptosis in endothelial and myocardial cells, HUVECs and H9C2, with induction by cytokines mix (CM) of IL-1 $\beta$ and IFN- $\gamma$.

CCK8 assay showed that HUVECs proliferation was dose-dependently increased compared to mimic control with transfection of miR-939-5p mimic from 50 to $150 \mathrm{nM}$ (Fig. 2A). In contrast, miR-939-5p antagomirs ranging from 50 to $150 \mathrm{nM}$ significantly inhibited proliferation (Fig. 2B). Further, miR-939-5p mimic elevated apoptosis inhibiting proteins expression of Bcl2 and CyclinB1 in HUVECs under CM treatment. While the apoptosis promoting proteins Bax and caspase 3 decreased obviously (Fig. 2C). Contrary, miR-939-5p antagomir resulted in down-regulation of Bcl2 and CyclinB1 and up-regulation of Bax and caspase 3 (Fig. 2D). Moreover, flow cytometry by annexin V-FITC/PI staining showed that miR-939-5p mimic inhibited CM induced HUVECs apoptosis in 0, 24, 48 and 60 hours with most significance in 48 hours (Fig. 2E), and miR-939-5p antagomir facilitated the CM induced HUVECs apoptosis from 0 to 60 hours with most typically in 48 hours (Fig. 2F), confirming the regulatory effects of miR-939-5p on inflammation induced cell apoptosis.

Similarly, we observed that miR-939-5p mimic promoted and antagomir inhibited H9C2 myocytes proliferation by CCK8 assay (Fig. supplementary1A), and miR-939-5p regulated apoptosis of H9C2 cardiomyocytes the same as in HUVECs by western blot (Fig. supplementary1B) and FACS analysis (Fig. supplementary1C).

Taken together, miR-939-5p could inhibit inflammation induced endothelial and myocardial cells apoptosis. 


\section{Identification of iNOS as a target of miR-939-5p}

MiRNAs regulate gene expression to influence pathogenesis and biogenesis by inhibiting mRNA translation or promoting degradation [34]. Previous study suggested that iNOS was candidate target gene of miR-939-5p according to microarray and bioinformatics RNAhybrid analysis [27]. INOS was predicted as miR-939-5p target gene in hepatocytes [25].

INOS, inducible nitric oxide synthetase (also known as NOS2), activated dramatically by inflammation, induces massive NO synthesis which is critical in CHF progression [35]. Thus we confirmed whether iNOS could be regulated by miR-939-5p. The results showed dose-dependent down-regulation of iNOS mRNA in HUVECs by miR-939-5p mimic (Fig. 3A) and dose-dependent up-regulation of iNOS mRNA by miR-939-5p antagomir (Fig. 3B). Also the protein level of iNOS expression was regulated by miR-939-5p mimic and antagomir dose-dependently according to western blot (Fig. 3C, D). Further, the nitrite oxide release by Griess assay showed that miR-939-5p mimic inhibited NO release into culture supernatants both in HUVECs and H9C2 (Fig. 3E,G). While miR-939-5p anatogmir promoted NO release in a dose-dependent manner (Fig. 3F, H). These results demonstrated that miR-939-5p could regulate the target gene iNOS expression and the following NO synthesis.

\section{Identification of TNF $\alpha$ as another target of miR-939-5p}

Our microarray data showed reduced $\mathrm{TNF} \alpha \mathrm{mRNA}$ level in miR-939-5p mimic group, and TargetScan database predicts two conserved binding sites of miR-939-5p in TNF $\alpha$ 3'UTR. Meanwhile TNF $\alpha$ could promote the expression of iNOS in mouse heart [23]. 
Thus, we investigated whether TNF $\alpha$ was another target of miR-939-5p.

We cloned wide type 3'-UTR sequence containing the two conserved binding sites (TNF $\alpha-3$ 'UTR-WT) and mutant sequence with two mutated binding sites (TNF $\alpha-3$ 'UTR-MUT) to generate luciferase reporter constructs (Fig. 4A). TNF $\alpha-3$ 'UTR-WT or TNF $\alpha-3$ 'UTR-MUT luciferase plasmids were co-transfected with miR-939-5p mimic or control mimics into HUVECs. And results showed that miR-939-5p mimic reduced the luciferase activity of TNF $\alpha$ 3'UTR-WT by about $75 \%$ versus mimic control, while no inhibitory effect was observed in mutant TNF $\alpha$ 3'UTR luciferase plasmid (Fig. 4B). Then, increasing doses of miR-939-5p mimics or antagomirs were transfected into HUVECs. It was found that miR-939-5p mimic inhibited TNF $\alpha$ mRNA in a dose dependent manner (Fig. 4C). By contrast, miR-939-5p antagomir led to a dose-dependent increase of TNF $\alpha$ mRNA (Fig. 4D). Moreover, ELISA assay confirmed the reduced TNF $\alpha$ level in cell supernatant by miR-939-5p mimic (Fig. 4E) while the increasing TNF $\alpha$ secretion by antagomir (Fig. 4F). These results suggested that TNF $\alpha$ was another bona-fide target of miR-939-5p.

\section{Identification of candidate IncRNAs interacted with miR-939-5p}

LncRNAs could act as miRNAs sponges to regulate their expression and target genes [36]. Using our microarray and bioinformatics prediction by DIANA-lncBase, lncRNAMap, LncRNADisease and lncRNome database, we screened 10 lncRNAs as candidates regulating miR-939-5p (Fig. 5A). By RT-qPCR, 3 lncRNAs, RP6-24A23.7, DMPK 3'UTR, NOS2P3, were selected to be reduced by miR-939-5p mimic and increased by antagomir in HUVECs (Fig. 5A), predicting possible interaction with 
miR-939-5p. For further confirmation, 3 lncRNAs were knocked down by smarter silencers. The knockdown efficiency of each silencer was detected by RT-qPCR (Fig. 5B). Intriguingly, only the knockdown of lnc-NOS2P3 led to the elevated miR-939-5p expression (Fig. 5C). And when we dose-dependently decreased NOS2P3 level using silencer-lncNOS2P3 (Fig. 5D), miR-939-5p expression was increased in a dose dependent manner (Fig. 5E). To further verify the regulation of NOS2P3 to miR-939-5p, NOS2P3 wide type cDNA and mutant cDNA with two mutated conserved miR-939-5p binding sites were constructed into pIRES2-GFP vectors (Fig. $5 \mathrm{~F})$. The transfection of $800 \mathrm{ng}$ lncNOS2P3-WT significantly reduced miR-939-5p level, with no same change by $\operatorname{lncNOS} 2 \mathrm{P} 3$-mut (Fig. 5G). It suggested that lncRNA-NOS2P3 might regulate miR-939-5p expression by direct base-pair binding to miR-939-5p.

\section{LncRNA-NOS2P3 functions as miR-939-5p sponge RNA and regulated the}

\section{expression of target genes, iNOS and TNFa}

NOS2P3, Nitric Oxide Synthase 2 Pseudogene 3, is the pseudogene 3 and the Ensembl number is ENSG00000230528 (from GeneCards). It localizes in chromosome17p11.2, the same chromosome with iNOS (NOS2), while iNOS localizes in $17 \mathrm{q} 11.2$. It is one of the three pseudogenes located in chromosome 17 neighboring iNOS [37] and its function has not been investigated yet.

To verify direct binding of NOS2P3 to miR-939-5p, NOS2P3 cDNA was cloned into the downstream of luciferase construct Rluc-GV272, with mutant cDNA construct of two conserved binding sites generated (Fig. 6A). MiR-939-5p mimic significantly 
decreased lnc-NOS2P3-WT luciferase activity, but had no similar influence to lnc-NOS2P3-mut (Fig. 6B).

With knocking down of endogenous NOS2P3 by si-lnc NOS2P3, the level of TNF $\alpha$ mRNA induced by CM obviously decreased (Fig. 6C). Next, we overexpressed lncNOS2P3-WT and lncNOS2P3-mut in HUVECs dose-dependently (Fig. 6D), and found TNF $\alpha$ mRNA increased dose-dependently following treatment with increasing dose of lncNOS2P3-WT concentration, but no effect observed by $\operatorname{lncNOS} 2 \mathrm{P} 3-\mathrm{mut}$ (Fig. 6E). Similarly, iNOS mRNA expression was increased dose-dependently following treatment with increased dose of lncNOS2P3-WT plasmids, but not by lncNOS2P3-mut (Fig. 6F). It suggests that NOS2P3 and miR-939-5p have base pairing interaction, which could influence the expression of target genes iNOS and TNF $\alpha$.

\section{Lnc-NOS2P3 regulated inflammatory cytokines induced apoptosis through miR-939-5p}

Although NOS2P3 interacts with miR-939-5p and regulates its expression and target genes, whether NOS2P3 affected inflammation induced apoptosis regulated by miR-939-5p was unknown.

NOS2P3 was knocked down or overexpressed and then rescued with miR-939-5p antagomir or mimic. Silencer-lnc-NOS2P3 increased the proliferation of HUVECs, but miR-939-5p antagomir reversed this elevated cell proliferation (Fig. 7A). Further flow cytometry analysis showed that the knockdown of endogenous NOS2P3 significantly reduced the apoptosis induced by cytokines mix, but miR-939-5p 
antagomir rescued the reduction of apoptosis (Fig. 7B). Moreover, western blot confirmed that silencer-lnc-NOS2P3 upregulated the apoptosis inhibiting proteins $\mathrm{Bcl} 2$ and $\mathrm{Cyclin} \mathrm{B} 1$ and inhibited the apoptosis promoting proteins Caspase 3 and Bax, and miR-939-5p antagomir reversed the expression of these proteins (Fig. 7C).

Next, we transfected lnc-NOS2P3-WT and mutant plasmids lnc-NOS2P3-mut with miR-939-5p mimic to further confirm it. Lnc-NOS2P3-WT significantly reduced the proliferation of HUVECs, while miR-939-5p mimic rescued it. However, lnc-NOS2P3-mutant didn't change the proliferation of HUVECs (Fig. 8A). Flow cytometry displayed that lnc-NOS2P3-WT (WT) aggravated the apoptosis induced by cytokines mix compared to negative control plasmid (NC), which was reversed by miR-939-5p mimic. No significant change was observed by lnc-NOS2P3-mutant (Mut) (Fig. 8B). Western blot further verified lnc-NOS2P3-WT decreased the expression of apoptosis inhibiting proteins and increased apoptosis promoting proteins expression, which were reversed by miR-939-5p mimic, but the mutant had no obvious affection (Fig. 8C). Collectively, NOS2P3 could regulate the cells apoptosis induced by inflammatory cytokines through miR-939-5p.

\section{Discussion}

The early pathogenesis of CHF is abnormal genes expression induced by neurohormones and inflammation, and following is myocardial and endothelial cells apoptosis [38]. The attempt to detect and prohibit early inflammation after heart injury is critical for early diagnosis and treatment. Here, we explored abnormal miR-939-5p 
expression in various stages of $\mathrm{CHF}$ and analyzed its relation with BNP. Then we investigated the mechanism by which miR-939-5p inhibits cytokines induced myocardial and endothelial cells apoptosis according to target genes iNOS and TNF $\alpha$. Furthermore, lncRNA NOS2P3 was explored to regulate miR-939-5p expression as a sponge RNA and influence miR-939-5p and target gens expression, controlling the cytokines induced apoptosis.

We found that serum miR-939-5p level was elevated in CHF patients than normal controls, greater in NYHA I-II patients than in NYHA III-IV. Further, in NYHA I-II patients, the significant correlation between miR-939-5p and BNP was found, while not in NYHA III-IV. NYHA I-II patients normally have mild or no clinical symptoms and are always to be neglected [2]. Although BNP is the most common diagnostic marker, once massive plasma BNP was detected, patients always have entered into NYHA III grade or worse [2]. BNP performs well to roll out, but less well to role in. Moreover, it's unclear to select optimal cut points of BNP with respect for the influence of factors like age and renal function [39]. MiR-939-5p in serum specially increased highly in NYHA I-II patients, and it was not related to BNP in this phase, indicating the possibility to assist BNP in diagnosis of CHF. Of course, in future study it needs further verification on randomized controlled trial (RCT) with more patients.

Previous research showed a robust induction of miR-939-5p in primary human hepatocytes after LPS or cytokine mix stimulation [25]. And miR-939-5p may regulate pro-inflammatory genes [40]. Importantly, heart failure has extensive correlation between elevated serum pro-inflammatory cytokines and adverse clinical 
outcomes [41, 42], yet the causative role of inflammation in disease progression is not well defined. The initial insult of CHF is mediated by inflammatory cytokines induced apoptosis [5] and the interaction between apoptotic cardiomyocytes and the microvascular network of endothelial cells post-MI has been studied extensively [23]. Our study showed that miR-939-5p could influence cytokines induced apoptosis of myocardial and endothelial cells. The inhibition of endothelial cells apoptosis in CHF pathogenesis may protect the microvascular of heart. And the reduction of myocardial cells apoptosis could prevent the process of CHF.

Target genes iNOS and TNF $\alpha$ were found in this study and they are key factors in NO system and regulation of CHF process. When initial insult occurred, the initial inflammatory/cytokines of heart will induce expression of miR-939-5p, which controlled the low level of iNOS and TNF $\alpha$ in NYHA I-II stages which may induce myocardial and endothelial cells apoptosis [21]. Myocardial and endothelial cells apoptosis induced by NO was reduced and normal cardiac function was preserved. As CHF processing, more and more inflammatory cytokines erupted, miR-939-5p was down-regulated and was not enough to inhibit iNOS and TNF $\alpha$, which would result in massive iNOS activation and high concentration NO production. Then abundant myocardial and endothelial cells apoptosis will be induced by NO, the heart will enter into decompensation stage of NYHA III-IV with typical clinical symptoms.

The relative balance between pathological inflammatory pathways and tissue reparative processes (physiological inflammation) define the trajectory of $\mathrm{HF}$ development [43]. Single application of immunosuppressant didn't achieve the 
desired therapeutic effect on CHF [5]. Although the elevated TNF $\alpha$ leads to worsening $\mathrm{HF}$ [32], the net result of blockade of $\mathrm{TNF} \alpha$ using soluble receptor infusion (etanercept) or humanized neutralizing antibodies (infliximab) was negative [44, 45]. It is worth exploring new therapy focusing on modestly lowering TNF $\alpha$ levels. And human natural existing lncRNA-miRNA network may be a promising choice. MiR-939-5p-iNOS/TNF $\alpha$ signal provide a new strategy in reversing the pathological inflammatory for treatment of CHF.

LncRNAs participate in a wide range of diseases, such as nervous system diseases, cancer and cardiovascular diseases [46-48], also can function as ceRNA of the miRNAs influencing heart failure [49]. LncRNA-ROR promoted cardiac hypertrophy through miR-133 [50]. Downregulated lncRNA-GASL1 in CHF regulates cardiomyocyte apoptosis [51]. Interfering with lncRNA ANRIL expression reduces heart failure in rats with diabetes by inhibiting myocardial oxidative stress [52]. Lnc-NOS2P3, the pseudogene 3, localizes in the same chromosome 17 neighboring iNOS (NOS2). Its function has not been reported now. We selected that lnc-NOS2P3 could regulate miR-939-5p expression according to direct sequence binding as a sponge RNA. Further, NOS2P3 influenced miR-939-5p target gene iNOS and TNF $\alpha$, affecting inflammation induced apoptosis of myocardial and endothelial cells. The spatial adjacency of NOS2P3 and iNOS indeed provides the possibility of their functional interaction with each other, and possibility to miR-939-5p-iNOS/TNF $\alpha$ pathway.

There exists the possible that in early stage of CHF, NOS2P3 was lowly expressed to 
release miR-939-5p and inhibit the target gene iNOS and TNF $\alpha$ and following NO synthesis, which prohibited myocardial and endothelial apoptosis. To late stage of CHF, the increasing expression of NOS2P3 inhibited the level of miR-939-5p, and resulted in extensive NO synthesis and cells apoptosis. But it needs certification in our future research.Moreover, the expression of lnc-NOS2P3-mut didn't completely reverse the regulation to miR-939-5p compared to 1 ncNOS2P3-WT. Cause, in addition to acting as ceRNA, lncRNAs has been reported to modulate process of diseases through interacting with DNA or proteins [34]. Maybe there exists other possible regulation pathway.

Although tens of thousands of non-coding RNAs have been found in humans, lots of lncRNAs and miRNAs do not show high interspecies conservation [53]. Although research showed that LPS and CM injection could induce miR-939-5p expression, the iNOS expression could not be inhibited in mouse or rat [25]. And we failed to find a homologous sequence of NOS2P3 and miR-939-5p in mice and rats by lncRNA and miRNA databases. Thus, we didn't investigate the function of miR-939-5p and lnc-NOS2P3 in vivo yet. But besides sequence conservation, many studies have proved other factors of lncRNA conservation, such as structure, function, and expression syntenic loci [54-56]. Future in vivo validation will be performed in advanced mammalian.

In conclusion, this study found the elevation of miR-939-5p in CHF, greater in NYHA I-II patients than NYHA III-IV. And we found that the regulation of lnc-NOS2P3-miR-939-5p to iNOS/ TNF $\alpha$ pathway and NO synthesis could restrict 
inflammation induced myocardial and endothelial cells apoptosis, which may prohibit the process of CHF and offer a novel strategy for treatment of CHF.

\section{Conclusion}

We demonstrated that CHF patients exhibited elevated serum miR-939-5p especially in NYHA I-II grades. Lnc-NOS2P3-miR-939-5p-iNOS/TNF a pathway played an important role in regulating inflammatory cytokines induced cells apoptosis and provided a promising strategy for diagnosis and treatment of CHF.

\section{Availability of data and materials}

The datasets used during the study are available from the corresponding author on reasonable request.

\section{Abbreviations}

LncRNA long non-coding RNA

miRNAs microRNAs

CHF chronic heart failure

iNOS induced nitric oxide synthase

TNF a tumor necrosis factor a

NOS2P3 Nitric Oxide Synthase 2 Pseudogene 3

HUVEC Human umbilical vein endothelial cells

NYHA New York Heart Association

BNP B-type natriuretic peptide

\section{Acknowledgements}

Not applicable 


\section{Funding}

This work was supported by National Natural Science Foundation of China (81601838), Foundation of Shanghai Municipal Health Commission (20164Y0175), National Natural Science Foundation of China (81601407) and Project of Tongji University (22120180025).

\section{Author information}

Lieying Fan and Cuncun Chen were Correspondence authors.

\section{Affiliaations}

Department of Clinical Laboratory, Shanghai East Hospital, Tongji University School of Medicine, 150 Ji Mo Road, Shanghai 200120, People’s Republic of China

\section{Authors' contributions}

Study concept and design: Cuncun Chen, Lieying Fan; Data collection: Cuncun Chen, Ming Zong, Ying Lu; Data analysis: Cuncun Chen, Yide Guo, Honggen Lv, Lihong Xie, Zhiyan Fu; Solving the experimental technique problems: Cuncun Chen, Yu Cheng, Yuying Si, Bei Ye; Manuscript writing: Cuncun Chen.

\section{Competing interests}

The authors declare that they have no competing interests.

\section{Ethics declarations}

\section{Ethics approval and consent to participate}

The Institutional Review Board of Tongji Medical School affiliated Shanghai East Hospital approved the study protocol and the written informed consent was obtained from each participant before any sample or data collection. 


\section{Consent for publication}

Not applicable

\section{Competing interests}

The authors declare that they have no competing interests.

\section{Additional information}

\section{Publisher's Note}

Springer Nature remains neutral with regard to jurisdictional claims in published maps and institutional affiliations. 


\section{References:}

1. Martinez F, Perna E, Perrone SV, Liprandi AS: Chagas Disease and Heart Failure: An Expanding Issue Worldwide. Eur Cardiol 2019, 14(2):82-88.

2. Yancy CW, Jessup M, Bozkurt B, Butler J, Casey DJ, Drazner MH, Fonarow GC, Geraci SA, Horwich T, Januzzi JL et al: 2013 ACCF/AHA guideline for the management of heart failure: a report of the American College of Cardiology Foundation/American Heart Association Task Force on Practice Guidelines. J AM COLL CARDIOL 2013, 62(16):e147-e239.

3. Hoshijima M, Chien KR: Mixed signals in heart failure: cancer rules. J CLIN INVEST 2002, 109(7):849-855.

4. Tanai E, Frantz S: Pathophysiology of Heart Failure. COMPR PHYSIOL 2015, 6(1):187-214.

5. Dick SA, Epelman S: Chronic Heart Failure and Inflammation: What Do We Really Know? CIRC RES 2016, 119(1):159-176.

6. Adrian L, Werner C, Laufs U: [ESC Guidelines 2016 - Heart Failure]. Dtsch Med Wochenschr 2017, 142(15):1123-1127.

7. Laflamme MA, Murry CE: Heart regeneration. NATURE 2011, 473(7347):326-335.

8. D'Uva G, Aharonov A, Lauriola M, Kain D, Yahalom-Ronen Y, Carvalho S, Weisinger K, Bassat E, Rajchman D, Yifa $\mathrm{O}$ et al: ERBB2 triggers mammalian heart regeneration by promoting cardiomyocyte dedifferentiation and proliferation. NAT CELL BIOL 2015, 17(5):627-638.

9. Pinto AR, Ilinykh A, Ivey MJ, Kuwabara JT, D'Antoni ML, Debuque R, Chandran A, Wang L, Arora K, Rosenthal NA et al: Revisiting Cardiac Cellular Composition. CIRC RES 2016, 118(3):400-409.

10. Doll S, Dressen M, Geyer PE, Itzhak DN, Braun C, Doppler SA, Meier F, Deutsch MA, Lahm H, Lange $\mathrm{R}$ et al: Region and cell-type resolved quantitative proteomic map of the human heart. NAT COMMUN 2017, 8(1):1469.

11. Smart N: Prospects for improving neovascularization of the ischemic heart: Lessons from development. MICROCIRCULATION 2017, 24(1).

12. Marin-Juez R, Marass M, Gauvrit S, Rossi A, Lai SL, Materna SC, Black BL, Stainier DY: Fast revascularization of the injured area is essential to support zebrafish heart regeneration. Proc Natl Acad Sci U S A 2016, 113(40):11237-11242.

13. Das S, Goldstone AB, Wang H, Farry J, D'Amato G, Paulsen MJ, Eskandari A, Hironaka CE, Phansalkar R, Sharma B et al: A Unique Collateral Artery Development Program Promotes Neonatal Heart Regeneration. CELL 2019, 176(5):1128-1142.

14. Rinn JL, Chang HY: Genome regulation by long noncoding RNAs. ANNU REV BIOCHEM 2012, 81:145-166.

15. Wang C, Zhang C, Liu L, A X, Chen B, Li Y, Du J: Macrophage-Derived mir-155-Containing Exosomes Suppress Fibroblast Proliferation and Promote Fibroblast Inflammation during Cardiac Injury. MOL THER 2017, 25(1):192-204.

16. Chouvarine P, Legchenko E, Geldner J, Riehle C, Hansmann G: Hypoxia drives cardiac miRNAs and inflammation in the right and left ventricle. J Mol Med (Berl) 2019.

17. Jiang L, Qiao Y, Wang $\mathrm{Z}$, Ma X, Wang $\mathrm{H}$, Li J: Inhibition of microRNA-103 attenuates inflammation and endoplasmic reticulum stress in atherosclerosis through disrupting the PTEN-mediated MAPK signaling. J CELL PHYSIOL 2019.

18. Han P, Li W, Lin CH, Yang J, Shang C, Nuernberg ST, Jin KK, Xu W, Lin CY, Lin CJ et al: A 
long noncoding RNA protects the heart from pathological hypertrophy. NATURE 2014, 514(7520):102-106.

19. Wang JX, Zhang XJ, Li Q, Wang K, Wang Y, Jiao JQ, Feng C, Teng S, Zhou LY, Gong Y et al: MicroRNA-103/107 Regulate Programmed Necrosis and Myocardial Ischemia/Reperfusion Injury Through Targeting FADD. CIRC RES 2015, 117(4):352-363.

20. Viereck J, Kumarswamy R, Foinquinos A, Xiao K, Avramopoulos P, Kunz M, Dittrich M, Maetzig

$\mathrm{T}$, Zimmer $\mathrm{K}$, Remke $\mathrm{J}$ et al: Long noncoding RNA Chast promotes cardiac remodeling. SCI TRANSL MED 2016, 8(326):322r-326r.

21. Wang $\mathrm{P}, \mathrm{Ba} Z \mathrm{ZF}$, Chaudry IH: Nitric oxide. To block or enhance its production during sepsis? Arch Surg 1994, 129(11):1137-1142, 1142-1143.

22. Habib FM, Springall DR, Davies GJ, Oakley CM, Yacoub MH, Polak JM: Tumour necrosis factor and inducible nitric oxide synthase in dilated cardiomyopathy. LANCET 1996, 347(9009):1151-1155.

23. Funakoshi H, Kubota T, Machida Y, Kawamura N, Feldman AM, Tsutsui H, Shimokawa H, Takeshita A: Involvement of inducible nitric oxide synthase in cardiac dysfunction with tumor necrosis factor-alpha. Am J Physiol Heart Circ Physiol 2002, 282(6):H2159-H2166.

24. Satoh M, Nakamura M, Tamura G, Makita S, Segawa I, Tashiro A, Satodate R, Hiramori K: Inducible nitric oxide synthase and tumor necrosis factor-alpha in myocardium in human dilated cardiomyopathy. $J$ AM COLL CARDIOL 1997, 29(4):716-724.

25. Guo Z, Shao L, Zheng L, Du Q, Li P, John B, Geller DA: miRNA-939 regulates human inducible nitric oxide synthase posttranscriptional gene expression in human hepatocytes. Proc Natl Acad Sci U S A 2012, 109(15):5826-5831.

26. Zhang X, Chen $\mathrm{C}$, Wu M, Chen L, Zhang J, Zhang X, Zhang Z, Wu J, Wang J, Chen X et al: Plasma microRNA profile as a predictor of early virological response to interferon treatment in chronic hepatitis B patients. ANTIVIR THER 2012, 17(7):1243-1253.

27. Chen $\mathrm{C}$, Wu M, Zhang W, Lu W, Zhang M, Zhang Z, Zhang X, Yuan Z: MicroRNA-939 restricts Hepatitis $B$ virus by targeting Jmjd3-mediated and C/EBPalpha-coordinated chromatin remodeling. Sci Rep 2016, 6:35974.

28. Li J, Liu K, Liu Y, Xu Y, Zhang F, Yang H, Liu J, Pan T, Chen J, Wu M et al: Exosomes mediate the cell-to-cell transmission of IFN-alpha-induced antiviral activity. NAT IMMUNOL 2013, 14(8):793-803.

29. Ouda R, Onomoto K, Takahasi K, Edwards MR, Kato H, Yoneyama M, Fujita T: Retinoic acid-inducible gene I-inducible miR-23b inhibits infections by minor group rhinoviruses through down-regulation of the very low density lipoprotein receptor. $J$ BIOL CHEM 2011, 286(29):26210-26219.

30. Hammerer-Lercher A, Collinson P, van Dieijen-Visser MP, Pulkki K, Suvisaari J, Ravkilde J, Stavljenic-Rukavina A, Baum H, Laitinen P: Do laboratories follow heart failure recommendations and guidelines and did we improve? The CARdiac MArker Guideline Uptake in Europe (CARMAGUE). CLIN CHEM LAB MED 2013, 51(6):1301-1306.

31. Zhang JX, Xu Y, Gao Y, Chen C, Zheng ZS, Yun M, Weng HW, Xie D, Ye S: Decreased expression of miR-939 contributes to chemoresistance and metastasis of gastric cancer via dysregulation of SLC34A2 and Raf/MEK/ERK pathway. MOL CANCER 2017, 16(1):18.

32. Mann DL: Innate immunity and the failing heart: the cytokine hypothesis revisited. CIRC RES 2015, 116(7):1254-1268. 
33. Schulz R, Panas DL, Catena R, Moncada S, Olley PM, Lopaschuk GD: The role of nitric oxide in cardiac depression induced by interleukin-1 beta and tumour necrosis factor-alpha. $\mathrm{Br} J$ Pharmacol 1995, 114(1):27-34.

34. Bayoumi AS, Sayed A, Broskova Z, Teoh JP, Wilson J, Su H, Tang YL, Kim IM: Crosstalk between Long Noncoding RNAs and MicroRNAs in Health and Disease. INT J MOL SCI 2016, 17(3):356.

35. de Vera ME, Shapiro RA, Nussler AK, Mudgett JS, Simmons RL, Morris SJ, Billiar TR, Geller DA: Transcriptional regulation of human inducible nitric oxide synthase (NOS2) gene by cytokines: initial analysis of the human NOS2 promoter. Proc Natl Acad Sci U S A 1996, 93(3):1054-1059.

36. Tay Y, Rinn J, Pandolfi PP: The multilayered complexity of ceRNA crosstalk and competition. NATURE 2014, 505(7483):344-352.

37. Fagerberg L, Hallstrom BM, Oksvold P, Kampf C, Djureinovic D, Odeberg J, Habuka M, Tahmasebpoor S, Danielsson A, Edlund K et al: Analysis of the human tissue-specific expression by genome-wide integration of transcriptomics and antibody-based proteomics. MOL CELL PROTEOMICS 2014, 13(2):397-406.

38. Vasan RS, Sullivan LM, Roubenoff R, Dinarello CA, Harris T, Benjamin EJ, Sawyer DB, Levy D, Wilson PW, D'Agostino RB: Inflammatory markers and risk of heart failure in elderly subjects without prior myocardial infarction: the Framingham Heart Study. CIRCULATION 2003, 107(11):1486-1491.

39. Hill SA, Booth RA, Santaguida PL, Don-Wauchope A, Brown JA, Oremus M, Ali U, Bustamam A, Sohel N, McKelvie R et al: Use of BNP and NT-proBNP for the diagnosis of heart failure in the emergency department: a systematic review of the evidence. HEART FAIL REV 2014, 19(4):421-438.

40. McDonald MK, Ramanathan S, Touati A, Zhou Y, Thanawala RU, Alexander GM, Sacan A, Ajit SK: Regulation of proinflammatory genes by the circulating microRNA hsa-miR-939. Sci Rep 2016, 6:30976.

41. Torre-Amione G, Kapadia S, Lee J, Durand JB, Bies RD, Young JB, Mann DL: Tumor necrosis factor-alpha and tumor necrosis factor receptors in the failing human heart. CIRCULATION 1996, 93(4):704-711.

42. Edelmann F, Holzendorf V, Wachter R, Nolte K, Schmidt AG, Kraigher-Krainer E, Duvinage A, Unkelbach I, Dungen HD, Tschope $\mathrm{C}$ et al: Galectin-3 in patients with heart failure with preserved ejection fraction: results from the Aldo-DHF trial. EUR J HEART FAIL 2015, 17(2):214-223.

43. Van Linthout S, Tschope C: Inflammation - Cause or Consequence of Heart Failure or Both? Curr Heart Fail Rep 2017, 14(4):251-265.

44. Mann DL, McMurray JJ, Packer M, Swedberg K, Borer JS, Colucci WS, Djian J, Drexler H, Feldman A, Kober L et al: Targeted anticytokine therapy in patients with chronic heart failure: results of the Randomized Etanercept Worldwide Evaluation (RENEWAL). CIRCULATION 2004, 109(13):1594-1602.

45. Chung ES, Packer M, Lo KH, Fasanmade AA, Willerson JT: Randomized, double-blind, placebo-controlled, pilot trial of infliximab, a chimeric monoclonal antibody to tumor necrosis factor-alpha, in patients with moderate-to-severe heart failure: results of the anti-TNF Therapy Against Congestive Heart Failure (ATTACH) trial. CIRCULATION 2003, 107(25):3133-3140.

46. Dangwal S, Schimmel K, Foinquinos A, Xiao K, Thum T: Noncoding RNAs in Heart Failure. 
Handb Exp Pharmacol 2017, 243:423-445.

47. Clark BS, Blackshaw S: Understanding the Role of IncRNAs in Nervous System Development. ADV EXP MED BIOL 2017, 1008:253-282.

48. Bhan A, Soleimani M, Mandal SS: Long Noncoding RNA and Cancer: A New Paradigm. CANCER RES 2017, 77(15):3965-3981.

49. Piccoli MT, Bar $\mathrm{C}$, Thum $\mathrm{T}$ : Non-coding RNAs as modulators of the cardiac fibroblast phenotype. J MOL CELL CARDIOL 2016, 92:75-81.

50. Jiang F, Zhou X, Huang J: Long Non-Coding RNA-ROR Mediates the Reprogramming in Cardiac Hypertrophy. PLOS ONE 2016, 11(4):e152767.

51. Deng H, Ouyang $\mathrm{W}$, Zhang $\mathrm{L}$, Xiao $\mathrm{X}$, Huang Z, Zhu W: LncRNA GASL1 is downregulated in chronic heart failure and regulates cardiomyocyte apoptosis. CELL MOL BIOL LETT 2019, 24:41.

52. Dai W, Lee D: Interfering with long chain noncoding RNA ANRIL expression reduces heart failure in rats with diabetes by inhibiting myocardial oxidative stress. J CELL BIOCHEM 2019.

53. Johnsson P, Lipovich L, Grander D, Morris KV: Evolutionary conservation of long non-coding RNAs; sequence, structure, function. Biochim Biophys Acta 2014, 1840(3):1063-1071.

54. Rogoyski OM, Pueyo JI, Couso JP, Newbury SF: Functions of long non-coding RNAs in human disease and their conservation in Drosophila development. Biochem Soc Trans 2017, 45(4):895-904.

55. Jenkins AM, Waterhouse RM, Muskavitch MA: Long non-coding RNA discovery across the genus anopheles reveals conserved secondary structures within and beyond the Gambiae complex. BMC GENOMICS 2015, 16:337.

56. Diederichs S: The four dimensions of noncoding RNA conservation. TRENDS GENET 2014, 30(4):121-123. 


\section{Figure legend}

Figure 1.The serum expression of miR-939-5p was higher in chronic heart failure patients than normal people. (A), The relative serum miR-939-5p expression was detected and compared in NYHA I-II grade, NYHA III-IV grade CHF patients and normal people by qRT-PCR, adding cel-39-3p as external control. Data are mean \pm S.E.M. ${ }^{*} p<0.05$ and ${ }^{* *} \mathrm{p}<0.01$. B and $\mathbf{C}$, The correlation between BNP and miR-939-5p level in CHF patients were analysed by Spearman's correlation. (B), there is no obvious correlation in CHF NYHA I-II patients, $(n=41) p>0.05$. (C), miR-939-5p expression in CHF NYHA III-IV patients was significantly correlated with BNP level, $(\mathrm{n}=24) \mathrm{r}=0.701, p<0.01$.

\section{Figure 2.MiR-939-5p dose-dependently regulated inflammation induced} apoptosis in HUVECs. A-D, miR-939-5p mimic or antagomir were transfected into HUVECs cells separately in 50nM, 100nM and $150 \mathrm{nM}$, treated with or without cytokines mix (CM) of $2 \mathrm{ng} / \mathrm{ml} \mathrm{IL-1 \beta}$ and $500 \mathrm{IU} / \mathrm{ml}$ IFN $\gamma$. (A), proliferation by CCK8 was dose-dependently upregulated by miR-939-5p mimics in blank or CM treatment, mimic control as negative control. (B), proliferation was dose-dependently down-regulated by miR-939-5p antagomir compared to antagomir control. (C), apoptosis proteins by western blot with miR-939-5p mimic and control. (D), apoptosis proteins by western blot with miR-939-5p antagomir and control. $\mathbf{E}$ and $\mathbf{F}$, effect of miR-939-5p on apoptosis by flow chart at different time points, $0 \mathrm{~h}, 24 \mathrm{~h}, 48 \mathrm{~h}$ and $60 \mathrm{~h}$. (E), miR-939-5p mimic vs. mimic control. (F), miR-939-5p antagomir vs. antagomir control. Data are mean \pm S.E.M. ${ }^{*} p<0.05$ and $^{* *} p<0.01$. 
Figure 3.INOS was the validated target gene of miR-939-5p. A-F, 50, 100 and 150nM miR-939-5p mimics or antagomirs were transfected into HUVECs with controls. The mRNA levels of iNOS by RT-qPCR in blank or CM treatment, with miR-939-5p mimics and control, GAPDH as internal control (A), or with miR-939-5p antagomirs and control (B). C and D, protein levels of iNOS with dose-dependently expression of miR-939-5p mimic or antagomir and controls, $\beta$-actin as internal control. (C), miR-939-5p mimic dose-dependently reduced protein level of iNOS. (D), knockdown of endogenous miR-939-5p dose dependently increased iNOS protein. E and $\mathbf{F}$, the concentration of nitric oxide in culture supernatant was detected using Griess assay (OD 540nm), (E), nitric oxide level elevated with dose-dependent expression of miR-939-5p mimics vs. control. (F), miR-939-5p antagomir reduced nitric oxide level. (G), nitric oxide level by Griess assay in $\mathrm{H} 9 \mathrm{C} 2$ cells was inhibited by miR-939-5p. (H), miR-939-5p antagomir raise the nitric oxide level in H9C2 cells. Data are mean \pm S.E.M. $* \mathrm{p}<0.05$ and $^{* *} \mathrm{p}<0.01$.

Figure 4.TNF $\alpha$ was another target gene of miR-939-5p. (A), putative miR-939-5p binding sites in the 3'UTR region of TNF $\alpha$ analyzed by TargetScan program. Mutated miR-939-5p binding sites (TNF $\alpha-3$ 'UTR-MUT) is shown. (B), luciferase constructs of TNF $\alpha-3$ 'UTR-WT or TNF $\alpha-3$ 'UTR-MUT were co-transfected into HUVECs with miR-939-5p mimic or control. PGL3 served as a control. The luciferase activity was analyzed. C and D, different concentration from 50-150 $\mathrm{nM}$ of miR-939-5p mimics 
or antagomirs were transfected into HUVECs. (C), miR-939-5p mimic reduced mRNA level of TNF $\alpha$. (D), miR-939-5p antagomir upregulated mRNA level of TNF $\alpha$. $\mathbf{E}$ and $\mathbf{F}, \mathrm{TNF} \alpha$ in the cell culture supernatant was detected by ELISA. (E), miR-939-5p mimic inhibited the level of TNF $\alpha$ vs. mimic control. (F), knockdown of endogenous miR-939-5p promoted TNF $\alpha$ secretion. Data are mean \pm S.E.M. ${ }^{*} p<0.05$ and $^{* *} p<0.01$.

Figure 5.Selection of candidate IncRNAs interacted with miR-939-5p. (A), 10 potential candidate lncRNAs from lncRNA-databases filtration of differentially expressed genes in lncRNA microarray. 100nM miR-939-5p mimic or antagomir and their controls were transfected into HUVECs. RNA was isolated and assayed by RT-qPCR, results are presented relative to GAPDH (2- $\left.2^{-\Delta \Delta C t}\right)$. B and C, 100nM smart silencers of $3 \operatorname{lncRNAs}($ si-lnc) were transfected into HUVECs. (B), the interference effects were quantified by qRT-PCR using lncRNAs specific primers from Ribobio, Guangzhou. (C), MiR-939-5p was measured by qRT-PCR, U6 as internal control. D and E, 50, 100, 150nM smart silencers of lnc-NO2P3 (si-lnc-NOS2P3) were transfected into HUVECs. (D), The level of lnc-NOS2P3 was determined. (E), Relevant miR-939-5p levels were detected versus U6. (F), lnc-NOS2P3 expression plasmid was constructed into pIRES2-EGFP. Lnc-NOS2P3-mut to two binding sites of miR-939-5p was formed. (G), 400ng and 800ng lnc-NO2P3-WT or lnc-NO2P3-mut and negative control were separately transfected into HUVECs, miR-939-5p levels were determined versus to U6. Data are mean 
\pm S.E.M. ${ }^{*} p<0.05$ and $^{* *} \mathrm{p}<0.01$.

Figure 6.Lnc-NOS2P3 was confirmed to regulate miR-939-5p and its target gene as sponge RNA. (A), lnc-NOS2P3 wide type and its mutant to miR-939-5p binding sites were cloned into luciferase carrier GV272 as lnc-NOS2P3-WT-luc and lnc-NOS2P3-mut-luc. (B), they were co-transfected into HUVECs with 100nM miR-939-5p mimic and control. Relative luciferase was obtained. (C), TNF $\alpha$ mRNA was obviously reduced after transfection of $100 \mathrm{nM}$ smart silencer of lnc-NOS2P3. D-F, 400ng and 800ng lnc-NOS2P3-WT or lnc-NOS2P3-mut GFP plasmid and their negative control were transfected into 24 plates with or without CM. (D), the increasing expression of lnc-NOS2P 3 and its mutant were detected by lnc-NOS2P 3 by RT-qPCR. GAPDH acted as control. (E), Relative TNF $\alpha$ mRNA level was measured 48 hours after treated with or without CM. (F), Relative iNOS mRNA level was obtained 48 hours later. Data are mean \pm S.E.M.* $p<0.05$ and $^{* *} p<0.01$.

Figure 7.Knockdown of endogenous Inc-NO2P3 inhibited the inflammation induced apoptosis which could be rescued by miR-939-5p antagomir. (A), 50nM or 100nM si-lnc-NOS2P3, 50nM or 100nM miR-939-5p antagomir (miR-939-5p an), si-negative control (si-N ctrl.), and antagomir control (An ctrl.) were transfected into HUVECs as groups shown. Cells proliferation was got by CCK8 assay. (B), Apoptosis cells were detected by flow cytometry in three replicate samples. The experiment was repeated twice and data were analyzed statistically. (C), Apoptosis related proteins 
were detected by western blot in various groups. Data are mean \pm S.E.M. $* p<0.05$ and ${ }^{*}$ p $<0.01$

Figure 8.Overexpression of Inc-NOS2P3 promoted inflammation induced apoptosis which was rescued by miR-939-5p mimic. (A), 800ng lnc-NOS2P3-WT or lnc-NOS2P3-mut and vector control were trasfected into HUVECs with 100nM miR-939-5p or mimic control. CCK8 assay showed its proliferation 48 hours later. B and C, 800ng lnc-NOS2P3-WT (WT) or lnc-NOS2P3-mut (Mut) and their vector control (NC) were transfected with 100nM miR-939-5p mimic (939mm) or its mimic control (mm). (B), Flow cytometry showed the apoptosis cells ratios, and data were analyzed statistically. (C), Apoptosis related proteins were measured by western blot. Data are mean \pm S.E.M. ${ }^{*} \mathrm{p}<0.05$ and $^{* *} \mathrm{p}<0.01$.

\section{Supplementary 1.MiR-939-5p regulated inflammation induced apoptosis in}

myocardial cells, H9C2. (A), 100nM miR-939-5p mimic was transfected into H9C2, cell vitality was assayed by CCK8. (B), 100nM miR-939-5p antagomir was transfected into $\mathrm{H} 9 \mathrm{C} 2$, cell vitality was assayed by CCK8, treated with or without CM. (C), Apoptosis related protein levels with miR-939-5p mimic were detected by western blot. (D), Protein levels with miR-939-5p antagomir were detected by western blot. (E), Apoptosis cells ratios with miR-939-5p mimic were detected by flow cytometry. (F), Apoptosis cells ratios with miR-939-5p mimic were detected by flow cytometry. Data are mean \pm S.E.M.* ${ }^{*}<0.05$ and $^{* *} p<0.01$. 
A

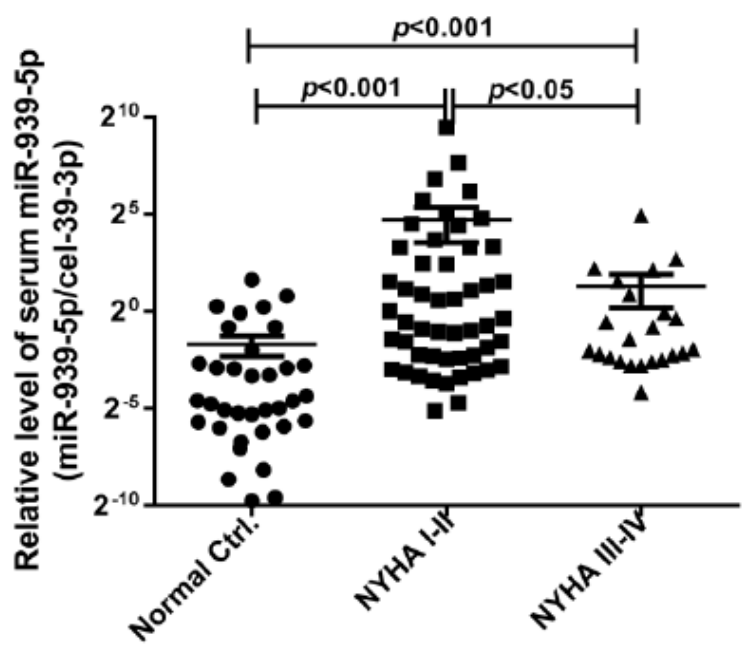

C

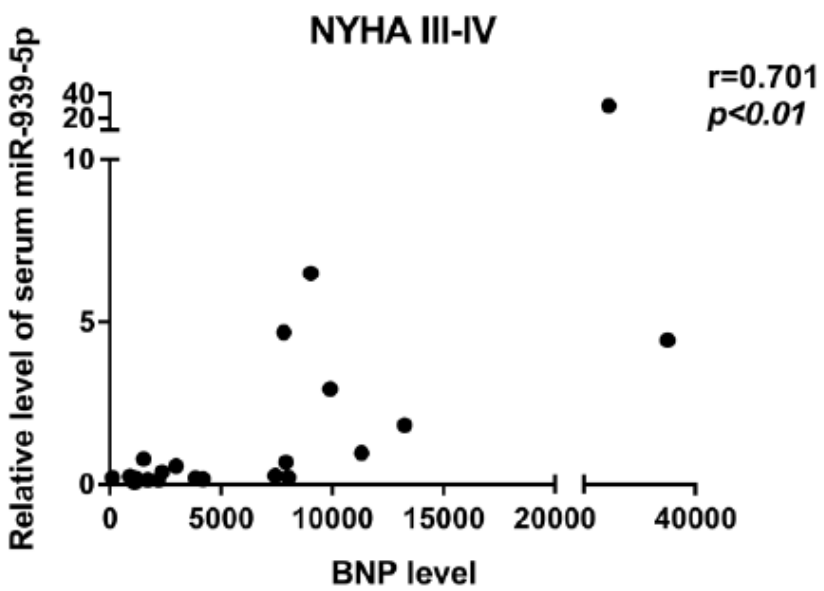

B

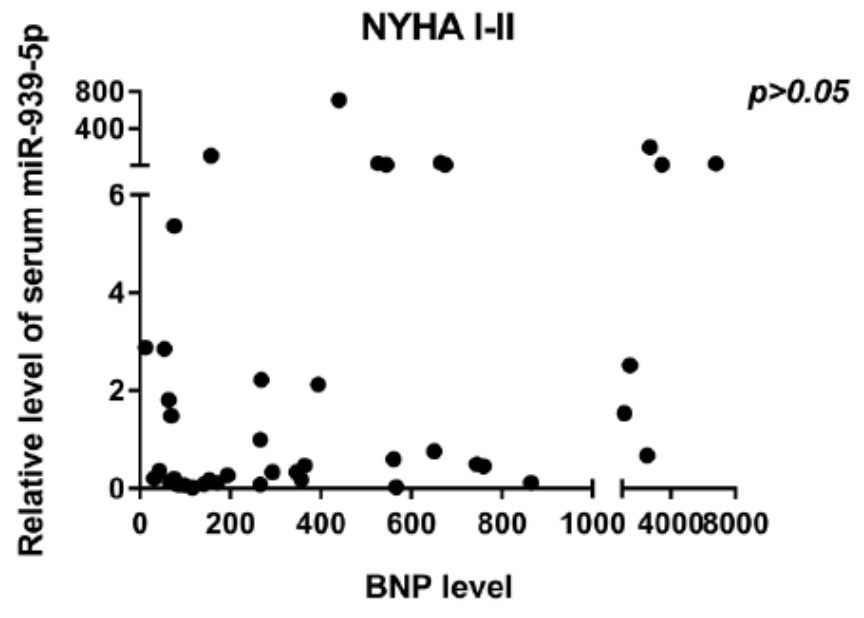

\section{Figure 1}

The serum expression of miR-939-5p was higher in chronic heart failure patients than normal people. (A), The relative serum miR-939-5p expression was detected and compared in NYHA I-II grade, NYHA III-IV grade CHF patients and normal people by qRT-PCR, adding cel-39-3p as external control. Data are mean \pm S.E.M. ${ }^{*} p<0.05$ and ${ }^{* \star} p<0.01$. B and C, The correlation between BNP and miR-939-5p level in CHF patients were analysed by Spearman's correlation. (B), there is no obvious correlation in CHF NYHA I-II patients, $(n=41) p>0.05$. (C), miR-939-5p expression in CHF NYHA III-IV patients was significantly correlated with BNP level, $(n=24) r=0.701, p<0.01$. 
A

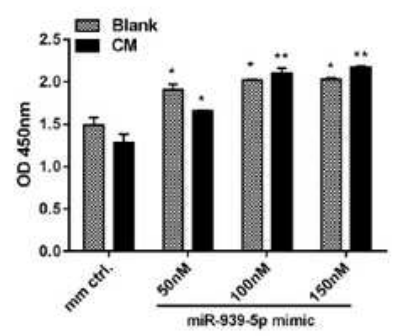

C
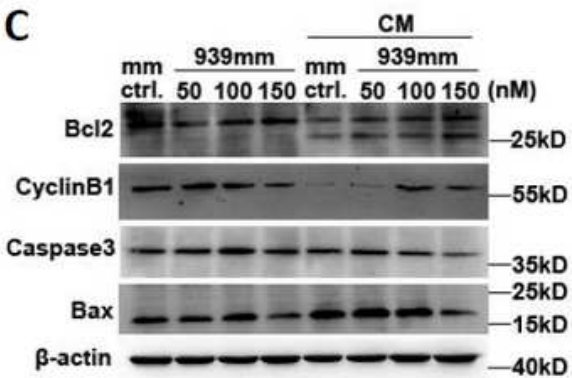

B

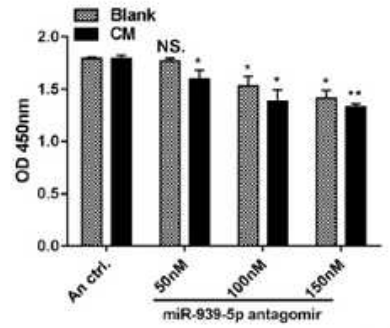

D

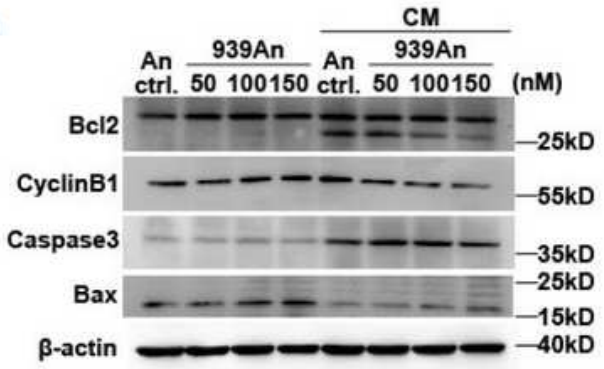

$60 \mathrm{~h}$

\section{E}

Oh

$24 h$

48h
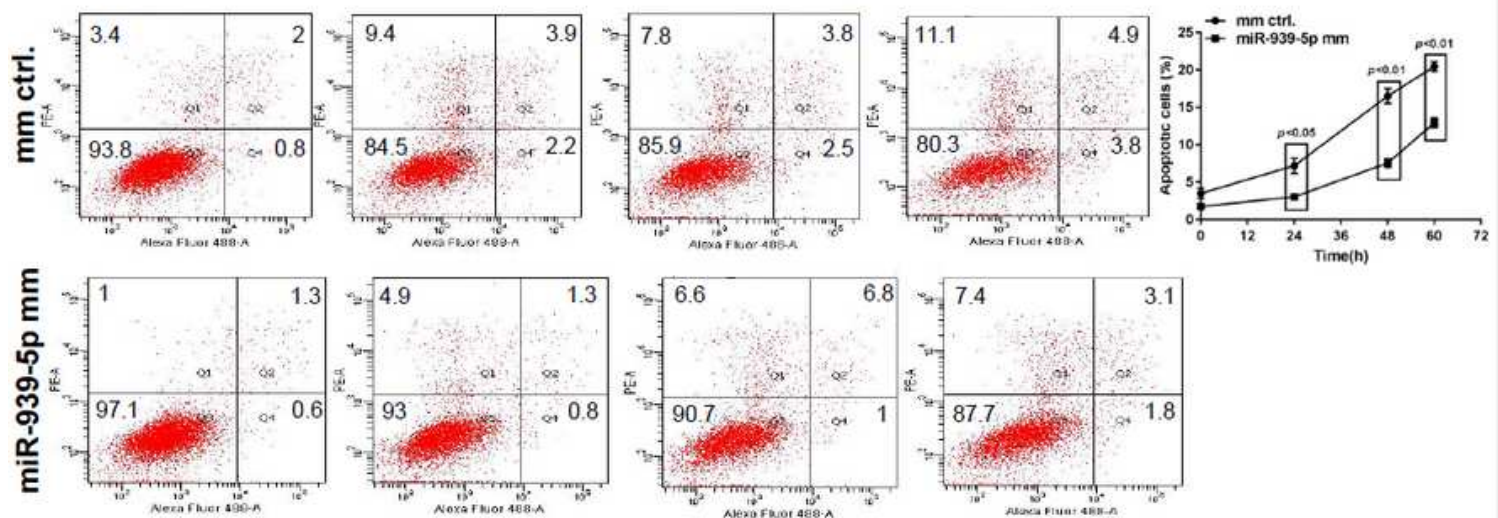

F

Oh

24h

48h

60h
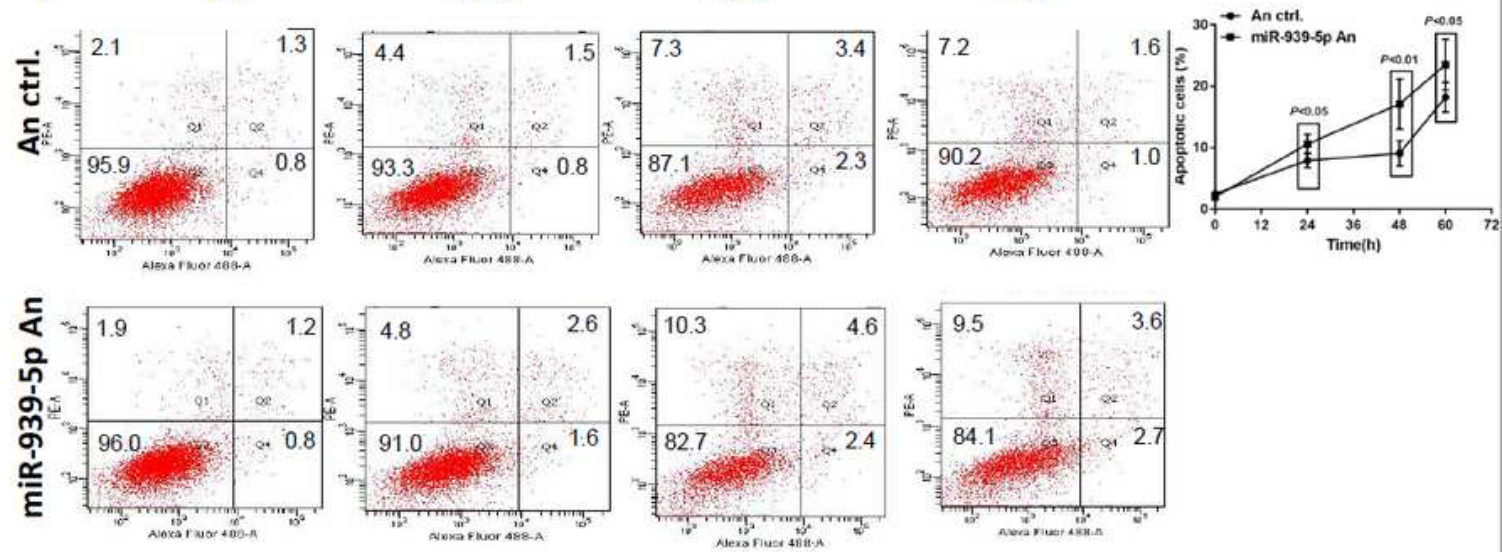

\section{Figure 2}

MiR-939-5p dose-dependently regulated inflammation induced apoptosis in HUVECs. A-D, miR-939-5p mimic or antagomir were transfected into HUVECs cells separately in $50 \mathrm{nM}, 100 \mathrm{nM}$ and $150 \mathrm{nM}$, treated with or without cytokines mix (CM) of $2 \mathrm{ng} / \mathrm{ml} \mathrm{IL-1 \beta}$ and $500 \mathrm{IU} / \mathrm{ml}$ IFNY. (A), proliferation by CCK8 was dose-dependently upregulated by miR-939-5p mimics in blank or $\mathrm{CM}$ treatment, mimic control as negative control. (B), proliferation was dose-dependently down-regulated by miR-939-5p antagomir compared to 
antagomir control. (C), apoptosis proteins by western blot with miR-939-5p mimic and control. (D), apoptosis proteins by western blot with miR-939-5p antagomir and control. E and F, effect of miR-939-5p on apoptosis by flow chart at different time points, $0 \mathrm{~h}, 24 \mathrm{~h}, 48 \mathrm{~h}$ and $60 \mathrm{~h}$. (E), miR-939-5p mimic vs. mimic control. (F), miR-939-5p antagomir vs. antagomir control. Data are mean \pm S.E.M. ${ }^{*} p<0.05$ and ${ }^{\star *} p<0.01$.

A

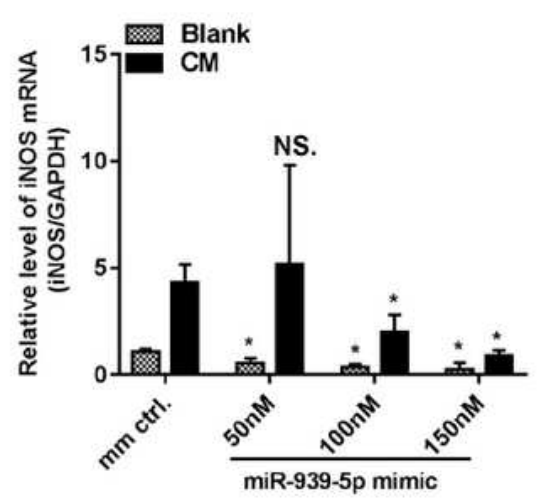

C

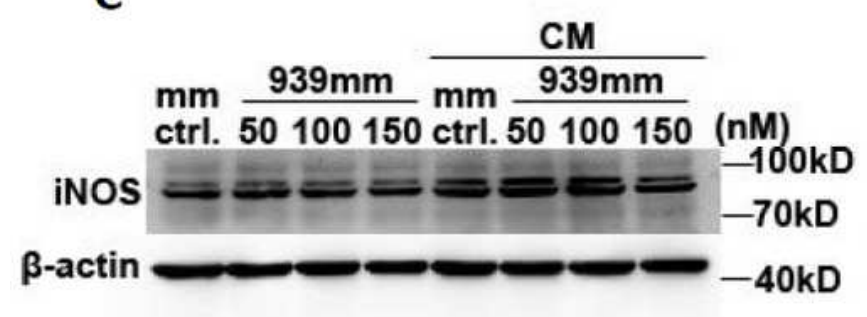

B

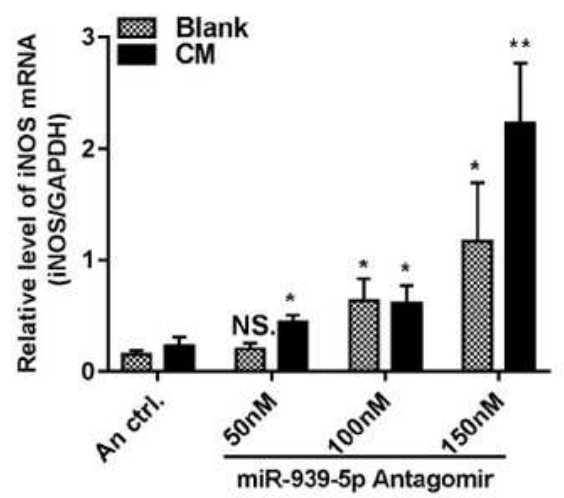

D

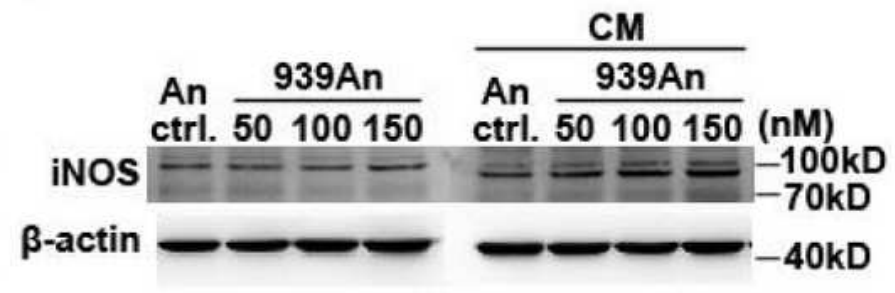

E

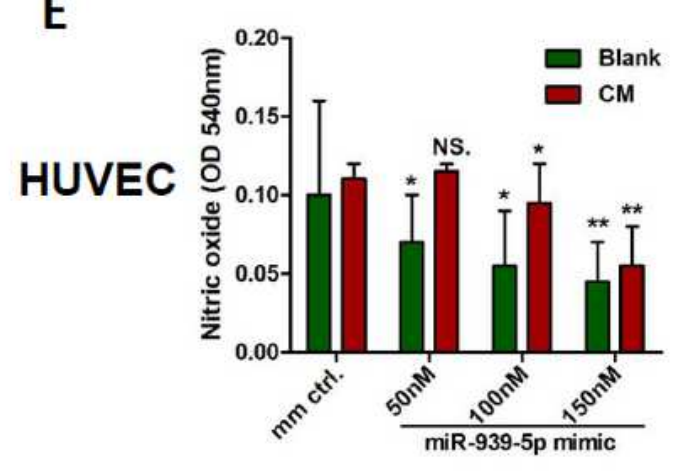

G

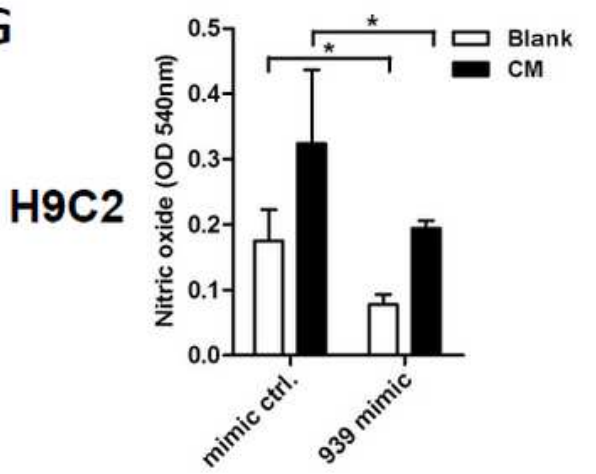

F

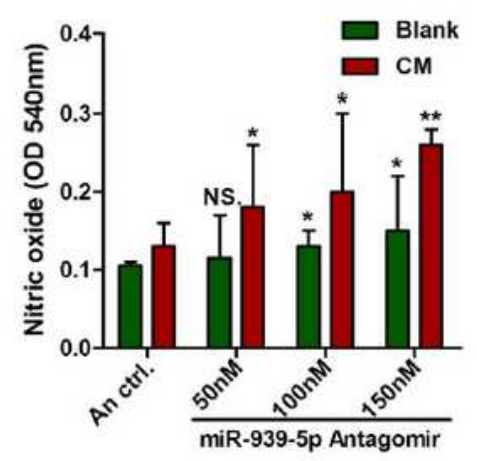

H

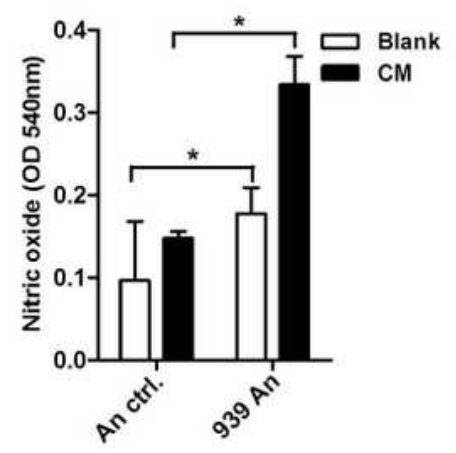

Figure 3 
INOS was the validated target gene of miR-939-5p. A-F, 50, 100 and 150nM miR-939-5p mimics or antagomirs were transfected into HUVECs with controls. The mRNA levels of iNOS by RT-qPCR in blank or CM treatment, with miR-939-5p mimics and control, GAPDH as internal control (A), or with miR-939-5p antagomirs and control (B). C and D, protein levels of iNOS with dose-dependently expression of miR-939$5 p$ mimic or antagomir and controls, $\beta$-actin as internal control. (C), miR-939-5p mimic dose-dependently reduced protein level of iNOS. (D), knockdown of endogenous miR-939-5p dose dependently increased iNOS protein. $E$ and $F$, the concentration of nitric oxide in culture supernatant was detected using Griess assay (OD 540nm), (E), nitric oxide level elevated with dose-dependent expression of miR-939-5p mimics vs. control. (F), miR-939-5p antagomir reduced nitric oxide level. (G), nitric oxide level by Griess assay in $\mathrm{H} 9 \mathrm{C} 2$ cells was inhibited by miR-939-5p. $(\mathrm{H})$, miR-939-5p antagomir raise the nitric oxide level in $\mathrm{H} 9 \mathrm{C} 2$ cells. Data are mean \pm S.E.M. ${ }^{\star} p<0.05$ and ${ }^{\star *} p<0.01$. 
A

Position 419-425 of TNFa 3'UTR 5'... ACACGGAGCCCAGCCCUCCCCAU.

hsa-miR-939-5p 3' GUGGGGGUCÚCGGAGUC--GAGGGGU

mutant of TNFa 3'UTR 5'...ACACGCUC GGCAGCCGAGGGGAU..

Position 726-733 of TNFa 3'UTR 5'...CAUUGCUGAGCCUCUGCUCCCCA...

hsa-miR-939-5p 3' GUGGCUCGGAGUCGAGGGGU

mutant of TNF $\alpha$ 3'UTR 5'...CAUUGCUCU ĆGGÁGU GGAGGGGA..

B

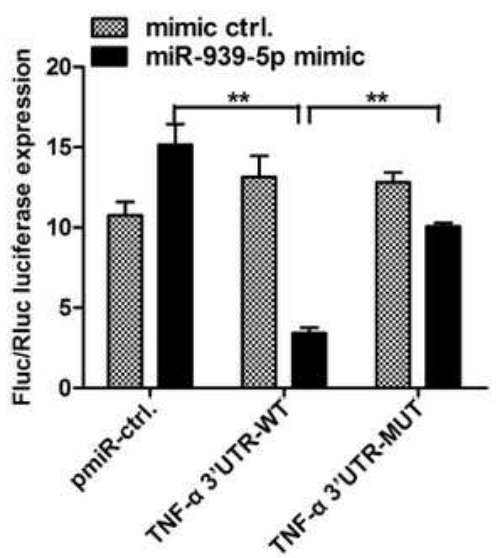

C

D
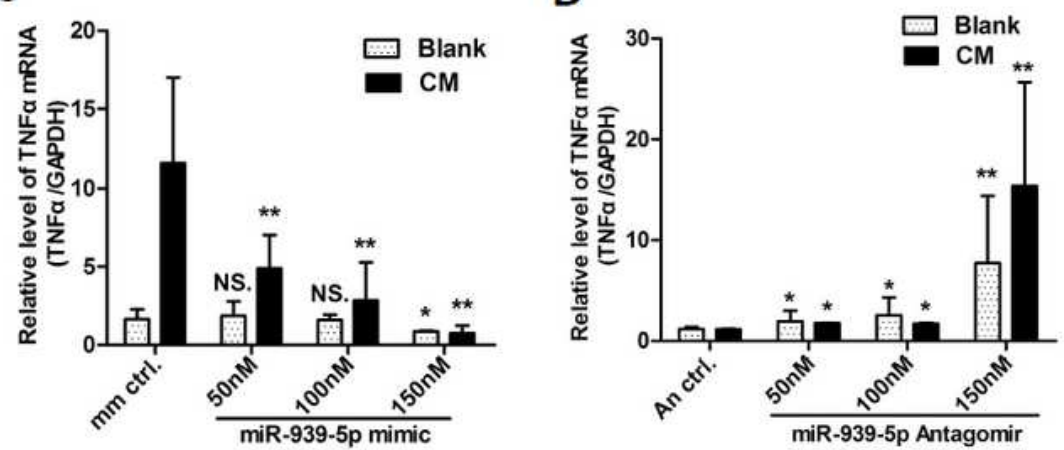

E

$\mathbf{F}$
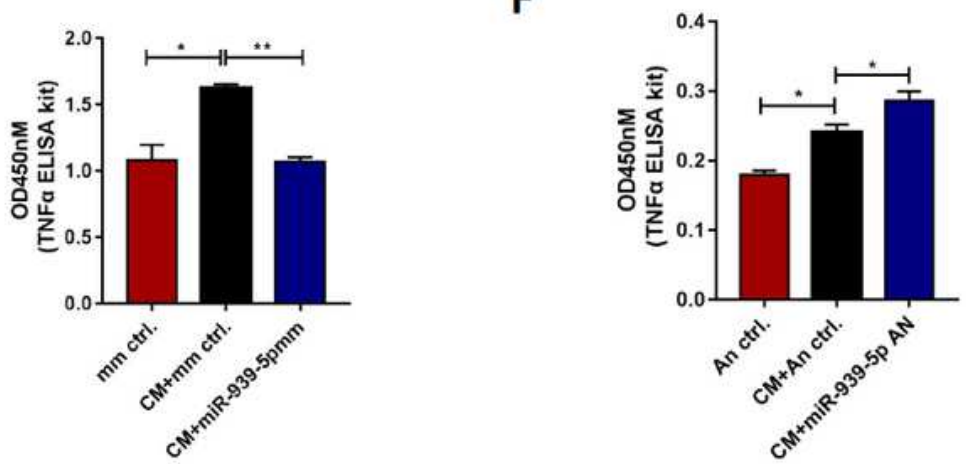

Figure 4

TNFa was another target gene of miR-939-5p. (A), putative miR-939-5p binding sites in the 3'UTR region of TNFa analyzed by TargetScan program. Mutated miR-939-5p binding sites (TNFa-3'UTR-MUT) is shown. (B), luciferase constructs of TNFa-3'UTR-WT or TNFa-3'UTR-MUT were co-transfected into HUVECs with miR-939-5p mimic or control. PGL3 served as a control. The luciferase activity was analyzed. C and D, different concentration from 50-150 nM of miR-939-5p mimics 32 or antagomirs were 
transfected into HUVECs. (C), miR-939-5p mimic reduced mRNA level of TNFa. (D), miR-939-5p antagomir upregulated mRNA level of TNFa. E and F, TNFa in the cell culture supernatant was detected by ELISA. (E), miR-939-5p mimic inhibited the level of TNFa vs. mimic control. (F), knockdown of endogenous miR939-5p promoted TNFa secretion. Data are mean \pm S.E.M. ${ }^{*} p<0.05 a n d^{* *} p<0.01$.
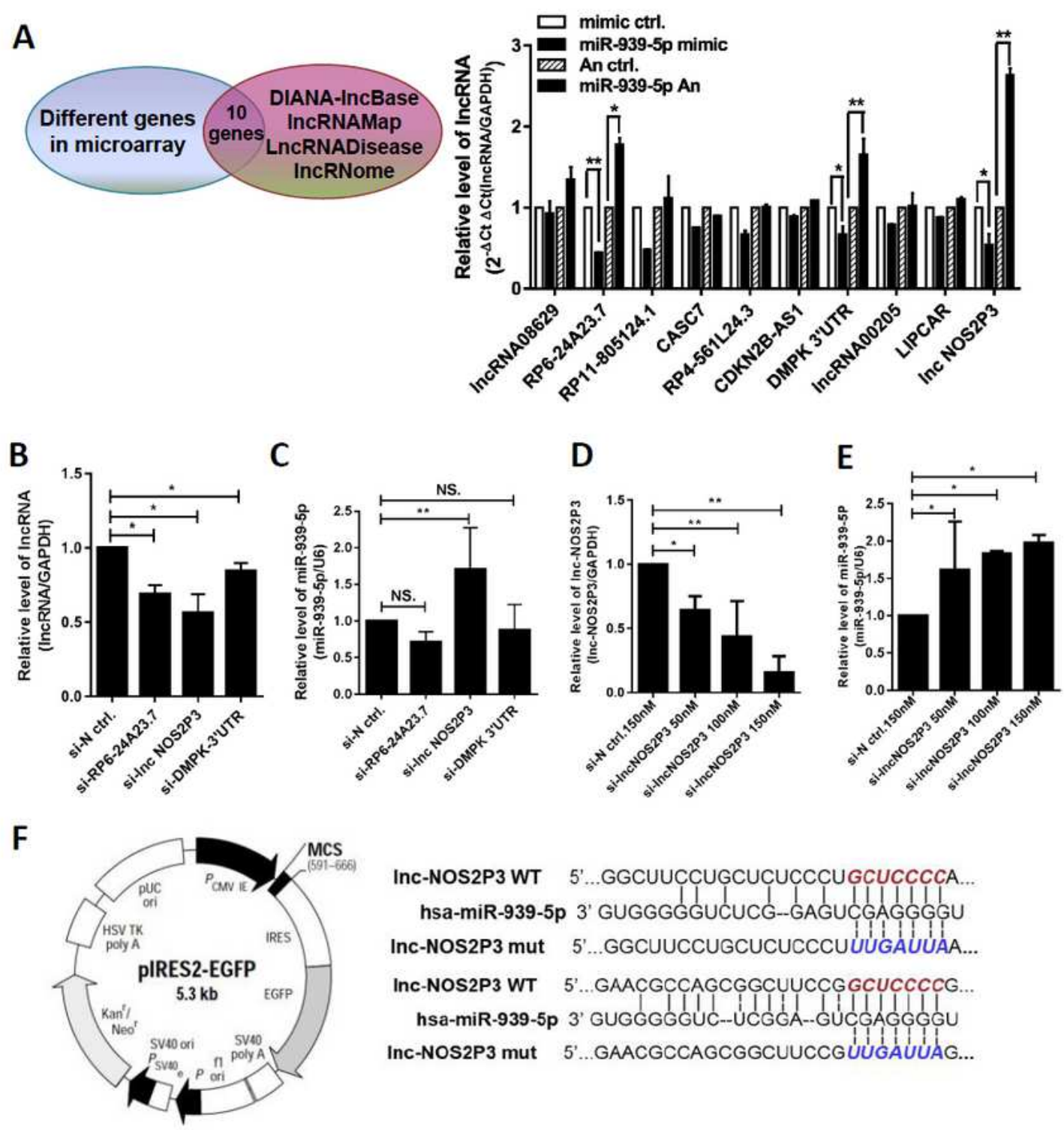

G

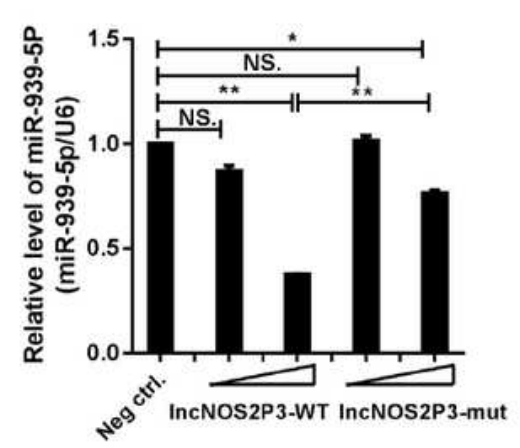

Figure 5 
Selection of candidate IncRNAs interacted with miR-939-5p. (A), 10 potential candidate IncRNAs from IncRNA-databases filtration of differentially expressed genes in IncRNA microarray. 100nM miR-939-5p mimic or antagomir and their controls were transfected into HUVECs. RNA was isolated and assayed by RT-qPCR, results are presented relative to GAPDH (2- $\Delta \Delta \mathrm{Ct})$. B and C, 100nM smart silencers of 3 IncRNAs (si-Inc) were transfected into HUVECs. (B), the interference effects were quantified by qRT-PCR using IncRNAs specific primers from Ribobio, Guangzhou. (C), MiR-939-5p was measured by qRT-PCR, U6 as internal control. D and E, 50, 100, 150nM smart silencers of Inc-NO2P3 (si-Inc-NOS2P3) were transfected into HUVECs. (D), The level of Inc-NOS2P3 was determined. (E), Relevant miR-939-5p levels were detected versus U6. (F), Inc-NOS2P3 expression plasmid was constructed into pIRES2-EGFP. Lnc-NOS2P3-mut to two binding sites of miR-939-5p was formed. (G), 400ng and 800ng Inc-NO2P3-WT or Inc-NO2P3-mut and negative control were separately transfected into HUVECs, miR-939-5p levels were determined versus to U6. Data are mean $33 \pm$ S.E.M. ${ }^{*}<<0.05$ and ${ }^{* *} p<0.01$. 
A

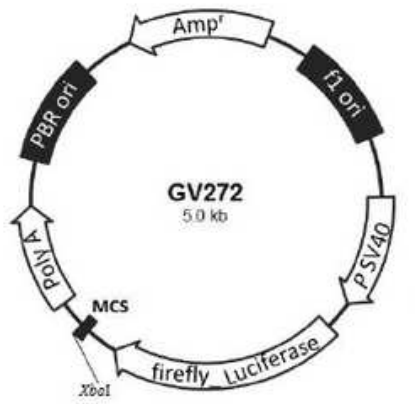

B

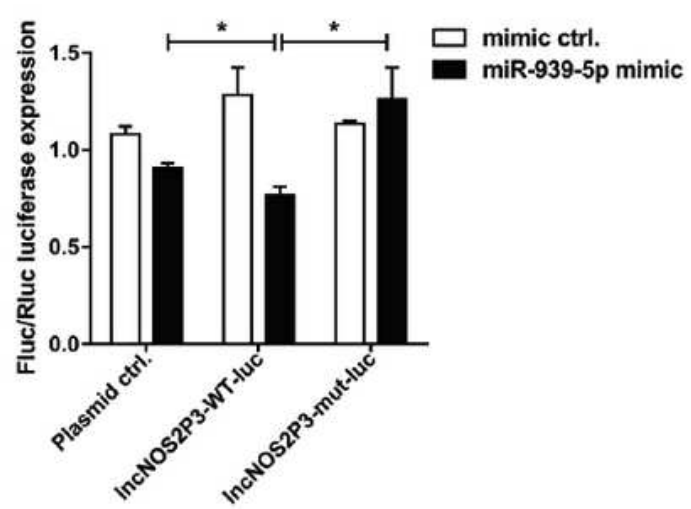

D

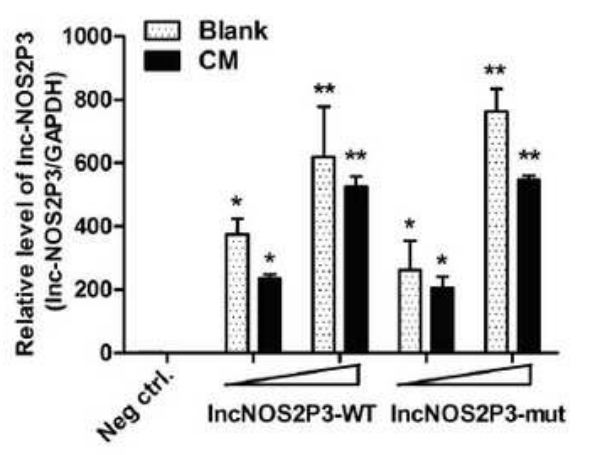

$\mathbf{F}$

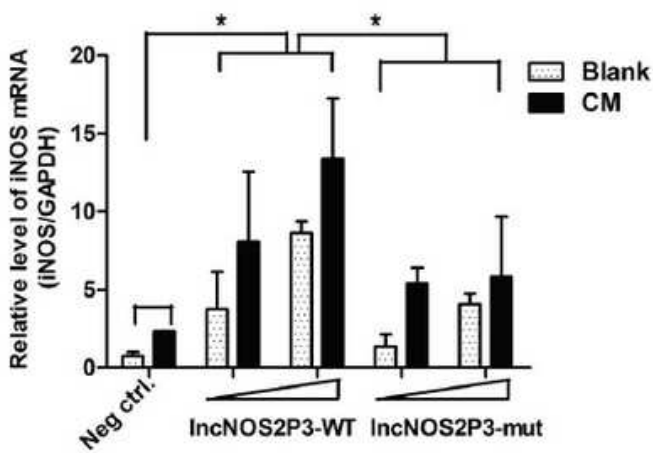

C

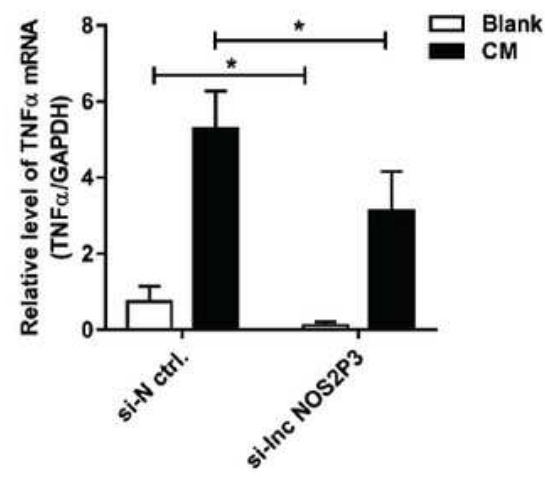

$\mathbf{E}$

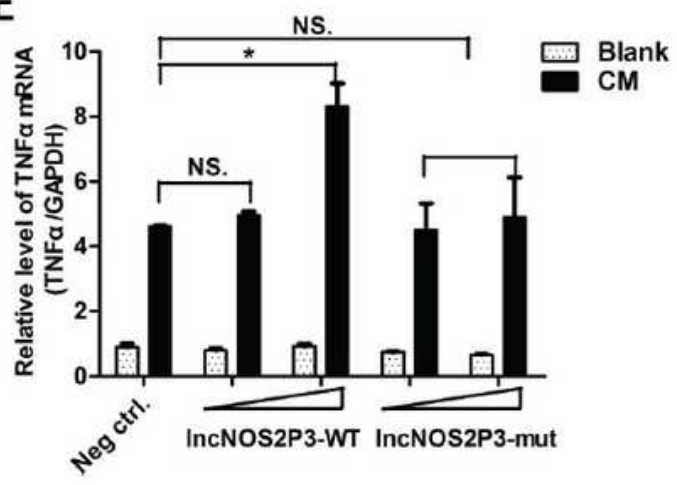

\section{Figure 6}

Lnc-NOS2P3 was confirmed to regulate miR-939-5p and its target gene as sponge RNA. (A), Inc-NOS2P3 wide type and its mutant to miR-939-5p binding sites were cloned into luciferase carrier GV272 as IncNOS2P3-WT-luc and Inc-NOS2P3-mut-luc. (B), they were co-transfected into HUVECs with 100nM miR939-5p mimic and control. Relative luciferase was obtained. (C), TNFa mRNA was obviously reduced after transfection of 100nM smart silencer of Inc-NOS2P3. D-F, 400ng and 800ng Inc-NOS2P3-WT or Inc- 
NOS2P3-mut GFP plasmid and their negative control were transfected into 24 plates with or without CM. (D), the increasing expression of Inc-NOS2P3 and its mutant were detected by Inc-NOS2P3 by RT-qPCR. GAPDH acted as control. (E), Relative TNFa mRNA level was measured 48 hours after treated with or without CM. (F), Relative iNOS mRNA level was obtained 48 hours later. Data are mean \pm S.E.M. ${ }^{\star} p<0.05$ and ${ }^{\star \star} p<0.01$.

A

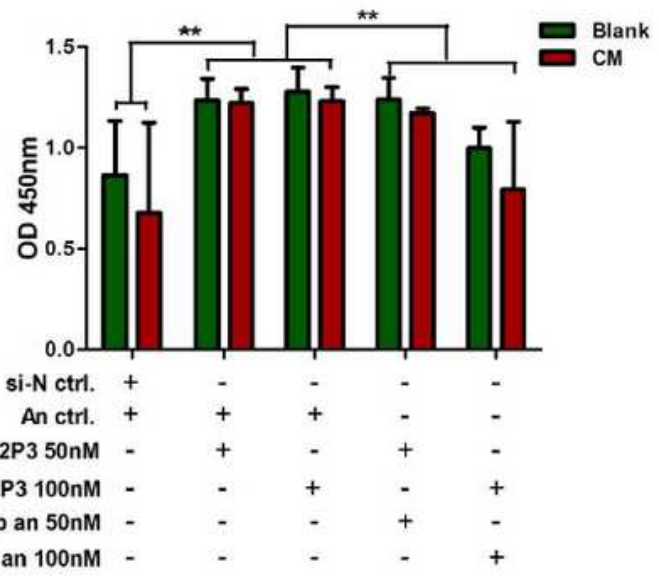

C
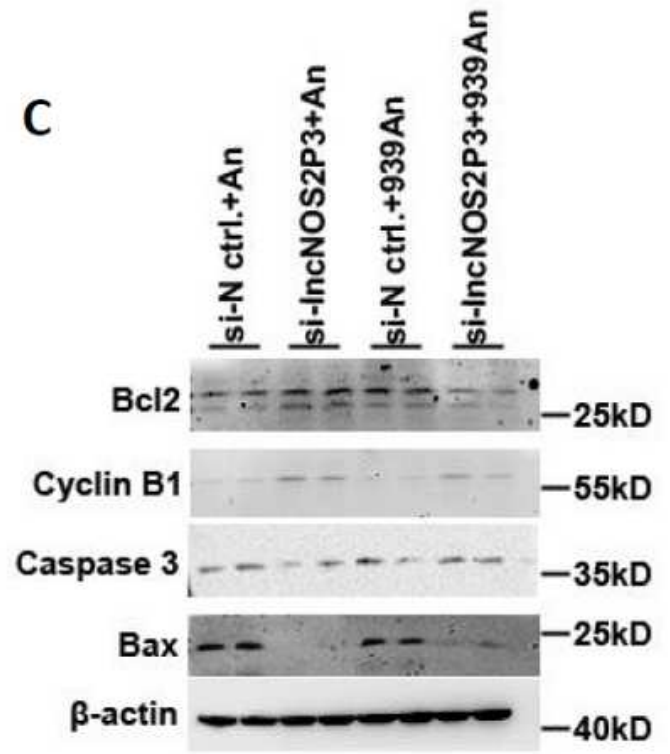

B

si-N ctrl.+An

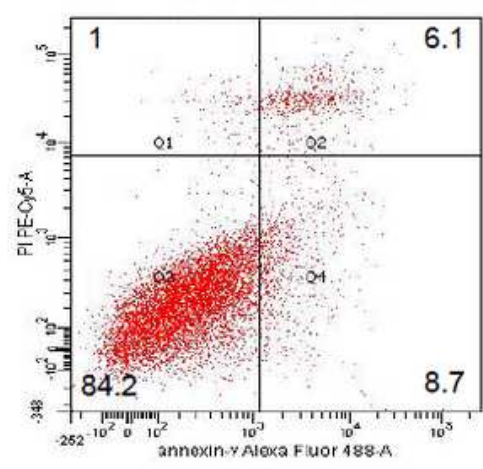

si-N ctrl.+miR-939-5p $\mathrm{An}+\mathrm{CM}$

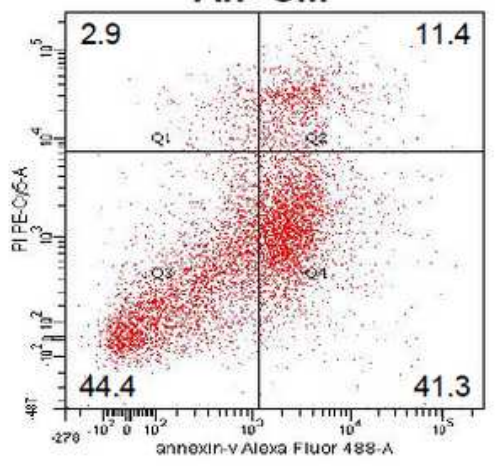

si-N ctrl. $+\mathrm{An}+\mathrm{CM}$

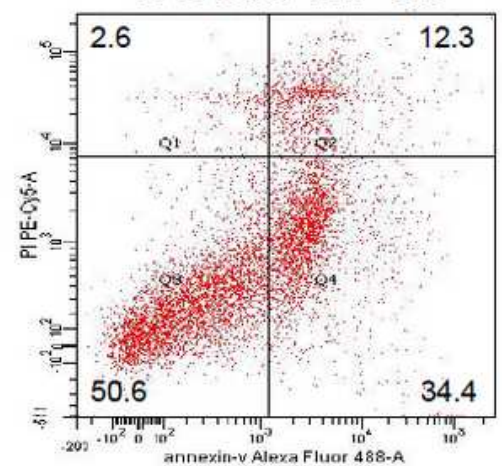

si-IncNOS2P3+miR-939-5p

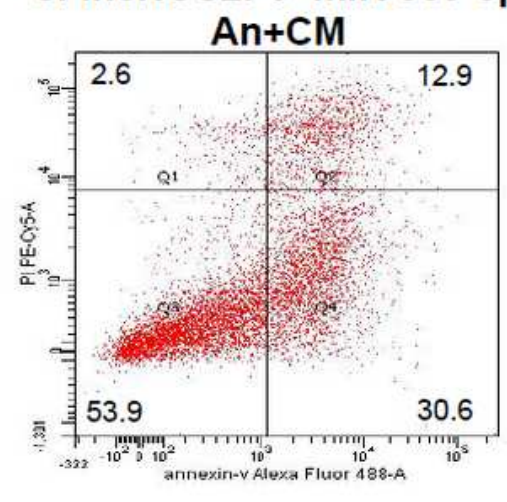

si-IncNOS2P $3+\mathrm{An}+\mathrm{CM}$
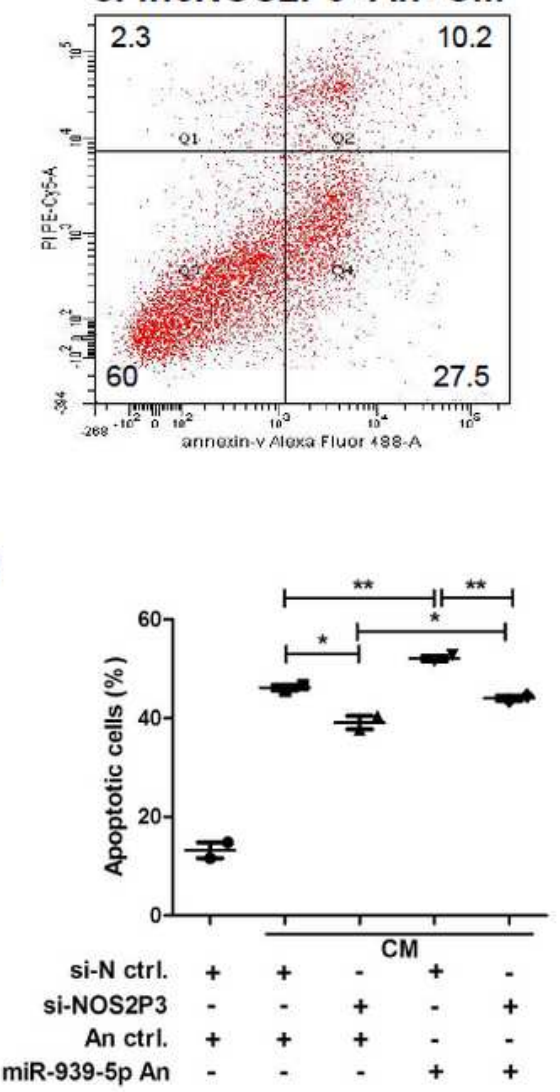

Figure 7 
Knockdown of endogenous Inc-NO2P3 inhibited the inflammation induced apoptosis which could be rescued by miR-939-5p antagomir. (A), 50nM or $100 \mathrm{nM}$ si-Inc-NOS2P3, 50nM or 100nM miR-939-5p antagomir (miR-939-5p an), si-negative control (si-N ctrl.), and antagomir control (An ctrl.) were transfected into HUVECs as groups shown. Cells proliferation was got by CCK8 assay. (B), Apoptosis cells were detected by flow cytometry in three replicate samples. The experiment was repeated twice and data were analyzed statistically. (C), Apoptosis related proteins 34 were detected by western blot in various groups. Data are mean \pm S.E.M. ${ }^{\star} p<0.05 a n d^{\star \star} p<<0.01$.

A
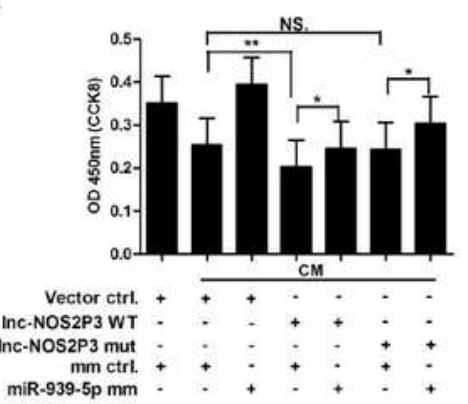

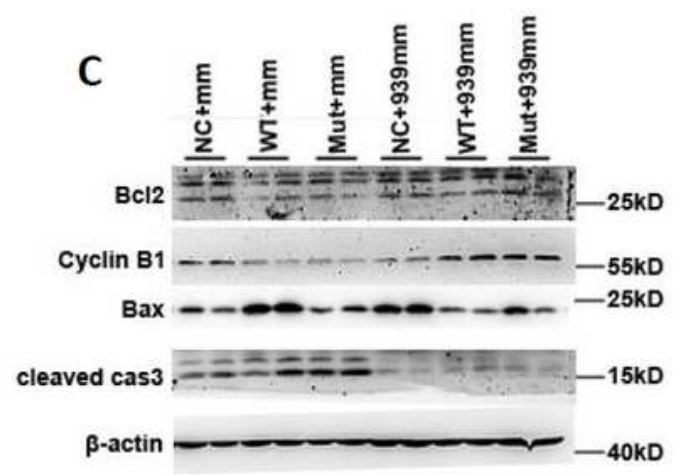

B
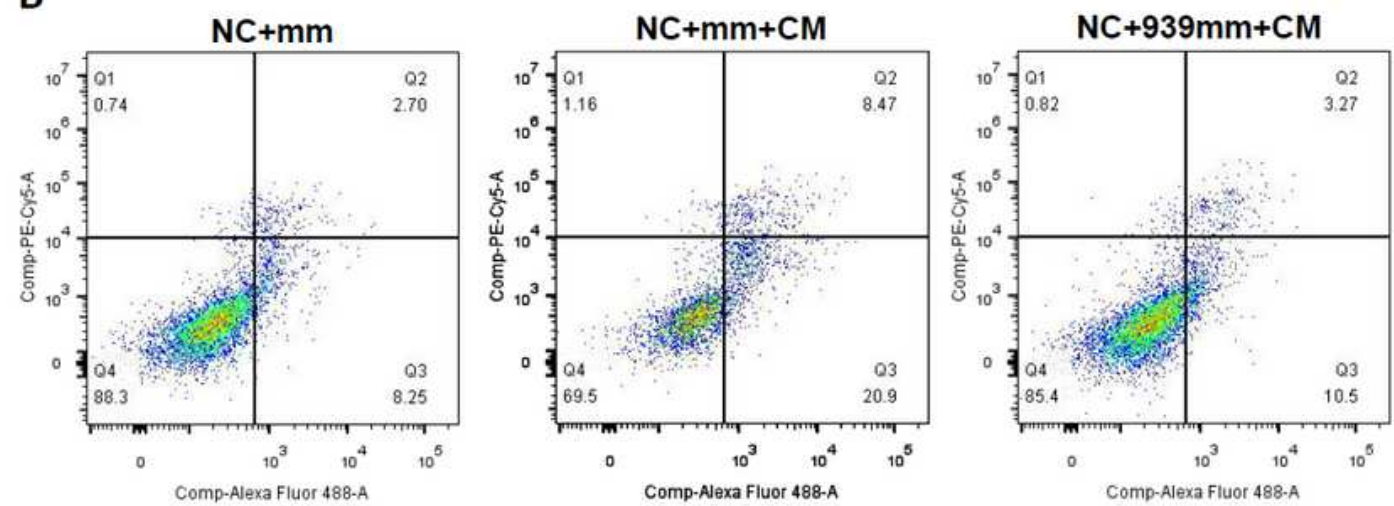

$\mathrm{WT}+\mathrm{mm}+\mathrm{CM}$

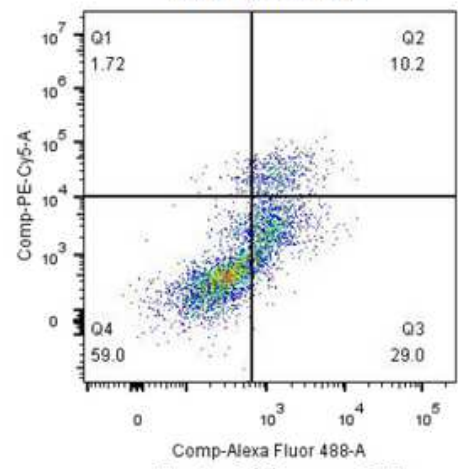

WT+939mm+CM

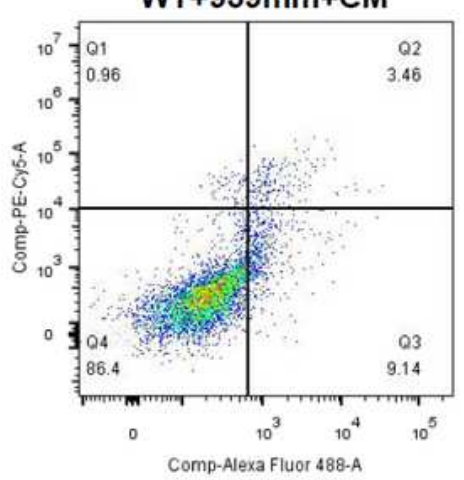

Mut+mm+CM
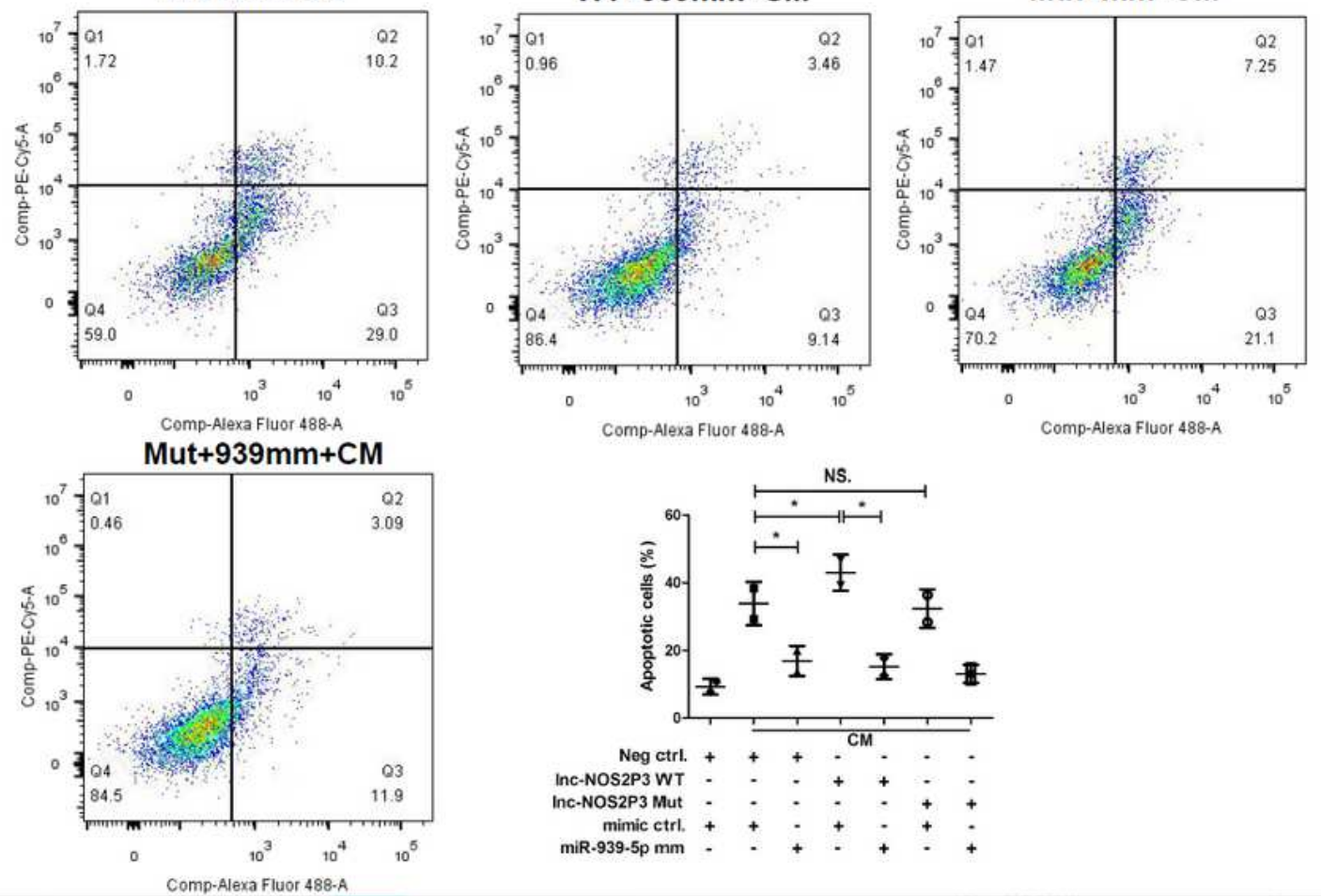


\section{Figure 8}

Overexpression of Inc-NOS2P3 promoted inflammation induced apoptosis which was rescued by miR939-5p mimic. (A), 800ng Inc-NOS2P3-WT or Inc-NOS2P3-mut and vector control were trasfected into HUVECs with 100nM miR-939-5p or mimic control. CCK8 assay showed its proliferation 48 hours later. B and C, 800ng Inc-NOS2P3-WT (WT) or Inc-NOS2P3-mut (Mut) and their vector control (NC) were transfected with $100 \mathrm{nM}$ miR-939-5p mimic $(939 \mathrm{~mm})$ or its mimic control $(\mathrm{mm})$. (B), Flow cytometry showed the apoptosis cells ratios, and data were analyzed statistically. (C), Apoptosis related proteins were measured by western blot. Data are mean \pm S.E.M. ${ }^{*} p<0.05 a n d * * p<0.01$.

\section{Supplementary Files}

This is a list of supplementary files associated with this preprint. Click to download.

- supplementary.pdf 